CALT-68-2407, DCPT/03/02

IPPP/03/01, LMU 18/02

TUM-HEP-481/02

hep-ph/0302174

\title{
The lightest Higgs Boson of mSUGRA, mGMSB and mAMSB at Present and Future Colliders: Observability and Precision Analyses
}

\author{
A. Dedes ${ }^{1 *}$, S. Heinemeyer ${ }^{2 \dagger}$, S. $\mathrm{Su}^{3 \ddagger}$ And G. Weiglein ${ }^{4 \S}$ \\ ${ }^{1}$ Physik Department, Technische Universität München, D-85748 Garching, Germany \\ ${ }^{2}$ Institut für theoretische Elementarteilchenphysik, LMU München, Theresienstr. 37, \\ D-80333 München, Germany \\ ${ }^{3}$ California Institute of Technology, Pasadena, CA 91125, USA \\ ${ }^{4}$ Institute for Particle Physics Phenomenology, University of Durham, \\ Durham DH1 3LE, UK
}

\begin{abstract}
We investigate the physics of the lightest $\mathcal{C} \mathcal{P}$-even MSSM Higgs boson at the Tevatron, the LHC, a linear $e^{+} e^{-}$collider, a $\gamma \gamma$ collider and a $\mu^{+} \mu^{-}$collider. The analysis is performed in the three most prominent soft SUSY-breaking scenarios, mSUGRA, mGMSB and mAMSB. For all colliders the observability and parameter regions with suppressed production cross sections (compared to a SM Higgs boson with the same mass) are investigated. For the lepton and photon colliders the potential is analyzed of precision measurements of the branching ratios of the light $\mathcal{C} \mathcal{P}$-even Higgs boson for obtaining indirect bounds on the mass of the $\mathcal{C P}$-odd Higgs boson and the highenergy parameters of the soft SUSY-breaking scenarios. In regions of the parameter space where the LHC can detect the heavy Higgs bosons, precision measurements of the properties of the light Higgs boson at the linear collider can provide valuable information for distinguishing between the mSUGRA, mGMSB and mAMSB scenarios.
\end{abstract}

*email: dedes@ph.tum.de

$\dagger$ †email: Sven.Heinemeyer@physik.uni-muenchen.de

‡email: shufang@theory.caltech.edu

§email: Georg.Weiglein@durham.ac.uk 


\section{Introduction}

The search for the light neutral Higgs boson is a crucial test of Supersymmetry (SUSY) that can be performed with the present and the next generation of high-energy colliders. The prediction of a relatively light Higgs boson is common to all supersymmetric models whose couplings remain in the perturbative regime up to a very high energy scale [1. Finding the Higgs boson and subsequently studying its couplings to fermions and bosons is thus one of the main goals of high-energy physics. The data taken during the final year of LEP running at $\sqrt{s} \gtrsim 206 \mathrm{GeV}$ have established a 95\% C.L. exclusion limit for the Standard Model (SM) Higgs boson of $M_{H_{\mathrm{SM}}}>114.4 \mathrm{GeV}$. They showed a slight excess at about the $2 \sigma$ level of signal-like events over the background expectation, which would be compatible with the expectation for the production of a Higgs boson with SM-like $Z Z H$ coupling with a mass $M_{H_{\mathrm{SM}}} \approx 116 \pm 1 \mathrm{GeV}$ [2]. In the Minimal Supersymmetric Standard Model (MSSM) the mass of the lightest $\mathcal{C P}$-even Higgs boson, $m_{h}$, is bounded from above by $m_{h} \lesssim 135 \mathrm{GeV}$ [3.4] (taking into account radiative corrections up to two-loop order [3,4,6,7,8,9,10,5,11,12,13,14]).

In the MSSM no specific assumptions are made about the underlying Supersymmetry(SUSY)-breaking mechanism, and a parameterization of all possible SUSY-breaking terms is used. This gives rise to the huge number of more than 100 new parameters in addition to the SM, which in principle can be chosen independently of each other. A phenomenological analysis of this model in full generality would clearly be very involved, and one usually restricts to certain benchmark scenarios, see e.g. Refs. [15, 16, 17. On the other hand, models in which all the low-energy parameters are determined in terms of a few parameters at the Grand Unification scale (or another high-energy scale), employing a specific soft SUSY-breaking scenario, are much more predictive. The most prominent scenarios in the literature are minimal Supergravity (mSUGRA) [18, 19], minimal Gauge Mediated SUSY Breaking (mGMSB) [20] and minimal Anomaly Mediated SUSY Breaking (mAMSB) [21, 22, 23. Analyses of the Higgs sector in these scenarios, mostly focusing only on the maximum value of $m_{h}$, have been performed in Refs. [24, 25, 26, 27, 28, 29, 30, 31]. A detailed comparison of the three soft SUSY-breaking scenarios in terms of exclusion regions in the $M_{A}-\tan \beta$-plane (where $A$ is the $\mathcal{C P}$-odd Higgs boson and $\tan \beta$ the ratio of the vacuum expectation values of the two Higgs doublets), their compatibility with the slight excess observed at LEP, and their corresponding SUSY particle spectra can be found in Ref. [32].

In the present paper the work of Ref. [32] is extended to an analysis of the lightest $\mathcal{C} \mathcal{P}$ even Higgs boson phenomenology at present and future colliders. We relate the input from the three soft SUSY-breaking scenarios in a uniform way to the predictions for the low-energy phenomenology in the Higgs sector, allowing thus a direct comparison of the predictions arising from the different scenarios. The high-energy parameters given in the three scenarios are related to the low-energy SUSY parameters via renormalization group (RG) running, taking into account contributions up to two-loop order 33. (for a recent comparison of different codes and current accuracies, see Ref. 34]). After transforming the parameters obtained in this way into the corresponding on-shell parameters 35, 36, 37, they are used as input for the program FeynHiggs 38, 39,40]. As a result the Higgs boson mass spectrum and the Higgs decay rates and branching ratios 41,42, have been obtained. Further restrictions such as from precision observables and the non-observation of SUSY particles at LEP and the Tevatron are also taken into account. For an analysis within the mSUGRA scenario where also the cold dark matter $(\mathrm{CDM})$ constraints are included, see Ref. [43]. Based on 
these predictions for the Higgs sector phenomenology, we analyze the observability of the lightest MSSM Higgs boson at the Tevatron, the LHC, a future $e^{+} e^{-}$linear collider (LC), a $\gamma \gamma$ collider $(\gamma \mathrm{C})$, and a $\mu^{+} \mu^{-}$collider $(\mu \mathrm{C})$. Regions of the high-energy parameter space with strongly suppressed Higgs production cross sections are identified. As the next step the branching ratios of the lightest Higgs boson into SM fermions $\left(h \rightarrow b \bar{b}, h \rightarrow c \bar{c}, h \rightarrow \tau^{+} \tau^{-}\right)$ and into $W$ bosons $\left(h \rightarrow W W^{*}\right)$ are compared for the mSUGRA, mGMSB and mAMSB case. We show that the precise measurement of the various Higgs decay branching ratios can be used to impose bounds on the value of $M_{A}$ and also the high-energy input parameters. Our analysis considerably differs from existing studies of Higgs boson branching ratios in the literature 44, 45, 46. In these previous analyses, all parameters except for the one under investigation (e.g. $M_{A}$ ) have been kept fixed and the effect of an assumed deviation between the MSSM and the SM has solely been attributed to this single free parameter. This would correspond to a situation with a complete knowledge of all other SUSY parameters without any experimental or theoretical uncertainty, which obviously leads to an unrealistic enhancement of the sensitivity to the investigated parameter. In our analysis we performed a more realistic study allowing all the SUSY parameters to vary. Furthermore, combined with the information on $M_{A}$ that could be obtained from the LHC Higgs searches, we discuss the possibility of distinguishing the three scenarios via the Higgs branching ratio measurements at the LC.

The rest of the paper is organized as follows. In Sect. 2 we briefly review the three soft SUSY-breaking scenarios and the evaluation of the low-energy data. The observability of the lightest MSSM Higgs boson is investigated in Sect. 3. In Sect. 4 the potential is analyzed of precision measurements of the Higgs boson branching ratios for obtaining indirect constraints on $M_{A}$ and the high-energy parameters of the soft SUSY-breaking scenarios. The possibility of a distinction of the mSUGRA, mGMSB and mAMSB scenarios is discussed. The conclusions can be found in Sect. 5.

\section{The low-energy sector and phenomenological con- straints}

In deriving the low-energy parameters in the three soft SUSY-breaking scenarios (mSUGRA, mGMSB and mAMSB) from the high-energy input parameters we follow Ref. [32. Thus, in this section only the most relevant facts are briefly summarized.

\subsection{The soft SUSY-breaking scenarios}

The fact that no SUSY partners of the SM particles have so far been observed means that low-energy SUSY cannot be realized as an unbroken symmetry in nature, and SUSY models thus have to incorporate additional Supersymmetry breaking interactions. This is achieved by adding to the Lagrangian (defined by the $\mathrm{SU}(3)_{C} \times \mathrm{SU}(2)_{L} \times \mathrm{U}(1)_{Y}$ gauge symmetry and the superpotential $W$ ) some further interaction terms that respect the gauge symmetry but break Supersymmetry (softly, i.e. no quadratic divergences appear), so called "soft SUSYbreaking" (SSB) terms. Assuming that the $R$-parity symmetry [47] is conserved, which we do in this paper for all SUSY breaking scenarios, reduces the amount of new soft terms allowed in the Lagrangian. Choosing a particular soft SUSY-breaking pattern allows further 
reduction of the number of free parameters and the construction of predictive models. The three most prominent scenarios for such models are

- mSUGRA (minimal Super Gravity scenario) [18, 19]:

Apart from the SM parameters (for the experimental values of the SM input parameters we use Ref. [48), 4 parameters and a sign are required to define the mSUGRA scenario:

$$
\left\{m_{0}, m_{1 / 2}, A_{0}, \tan \beta, \operatorname{sign}(\mu)\right\} .
$$

While $m_{0}, m_{1 / 2}$ and $A_{0}$ define the scalar and fermionic masses and the trilinear couplings at the GUT scale $\left(\sim 10^{16} \mathrm{GeV}\right), \tan \beta$ (the ratio of the two vacuum expectation values) and the $\operatorname{sign}(\mu)$ ( $\mu$ is the supersymmetric Higgs mass parameter) are defined at the low-energy scale. For our numerical analysis, see Sects. 3, 4, we have scanned over the following parameter space $^{1}$ :

$$
\begin{aligned}
& 50 \mathrm{GeV} \leq m_{0} \leq 1 \mathrm{TeV}, \\
& 50 \mathrm{GeV} \leq m_{1 / 2} \leq 1 \mathrm{TeV}, \\
& -3 \mathrm{TeV} \leq A_{0} \leq 3 \mathrm{TeV} \text {, } \\
& 1.5 \leq \tan \beta \leq 60, \\
& \operatorname{sign} \mu=+1 \text {. }
\end{aligned}
$$

The low-energy spectrum has been evaluated with the programs $S U I T Y / F e y n S S G$ [51, 52 .

- mGMSB (minimal Gauge Mediated SUSY-Breaking) [20]:

A very promising alternative to mSUGRA is based on the hypothesis that the soft SUSY-breaking occurs at relatively low energy scales and is mediated mainly by gauge interactions through the so-called "messenger sector" [54,53,20]. Also in this scenario, the low-energy parameters depend on 4 parameters and a sign,

$$
\left\{M_{\text {mess }}, N_{\text {mess }}, \Lambda, \tan \beta, \operatorname{sign}(\mu)\right\},
$$

where $M_{\text {mess }}$ is the overall messenger mass scale; $N_{\text {mess }}$ is a number called the messenger index, parameterizing the structure of the messenger sector; $\Lambda$ is the universal soft SUSY-breaking mass scale felt by the low-energy sector. The phenomenology of mGMSB is characterized by the presence of a very light gravitino $\tilde{G}$ with mass given by $m_{3 / 2}=m_{\tilde{G}}=\frac{F}{\sqrt{3} M_{P}^{\prime}} \simeq\left(\frac{\sqrt{F}}{100 \mathrm{TeV}}\right)^{2} 2.37 \mathrm{eV}$ [55], where $\sqrt{F}$ is the fundamental scale of SSB and $M_{P}^{\prime}=2.44 \times 10^{18} \mathrm{GeV}$ is the reduced Planck mass. Since $\sqrt{F}$ is typically of order $100 \mathrm{TeV}$, the $\tilde{G}$ is always the LSP in these theories. The numerical analysis in Sects. 3, 4 is based on the following scatter ranges:

$$
\begin{aligned}
10^{4} \mathrm{GeV} & \leq \Lambda \\
1.01 \Lambda & \leq 2 \times 10^{5} \mathrm{GeV} \\
1 \leq M_{\text {mess }} & \leq 10^{5} \Lambda, \\
1.5 & \leq \tan \beta \leq 60 \\
\operatorname{sign} \mu & =+1 .
\end{aligned}
$$

\footnotetext{
${ }^{1}$ The sign of $\mu$ has been fixed to $(+)$ (for all three soft SUSY-breaking scenarios), since this sign is favored by the $g_{\mu}-2$ [49] and the $\operatorname{BR}(b \rightarrow s \gamma)$ [50] constraints, see Sect. 2.3]
} 
The low-energy parameter sets for this scenario have been calculated by using the program SUSYFIRE [56] and adopting the phenomenological approach of Refs. [57, 58, 59, 29].

- mAMSB (minimal Anomaly Mediated SUSY-Breaking) [21,22, 23]:

In this model, SUSY breaking happens on a separate brane and is communicated to the visible world via the super-Weyl anomaly. The particle spectrum is determined by 3 parameters and a sign:

$$
\left\{m_{\text {aux }}, m_{0}, \tan \beta, \operatorname{sign}(\mu)\right\} .
$$

The overall scale of SUSY particle masses is set by $m_{\text {aux }}$, which is the VEV of the auxiliary field in the supergravity multiplet. $m_{0}$ is introduced as a phenomenological parameter to avoid negative slepton mass squares, for other approaches to this problem see Refs. $[21,60,61,62,63$. The scatter parameter space for the numerical analysis in Sects. 3, 4 is chosen to be

$$
\begin{aligned}
20 \mathrm{TeV} & \leq m_{\text {aux }} \leq 100 \mathrm{TeV} \\
0 \leq m_{0} & \leq 2 \mathrm{TeV} \\
1.5 \leq \tan \beta & \leq 60 \\
& \operatorname{sign} \mu=+1
\end{aligned}
$$

The low-energy spectrum has been derived with the code described in Ref. [26].

\subsection{Evaluation of predictions in the Higgs boson sector of the MSSM}

The most relevant parameters for Higgs boson phenomenology in the MSSM are the mass of the $\mathcal{C P}$-odd Higgs boson, $M_{A}$, the ratio of the two vacuum expectation values, $\tan \beta$, the scalar top masses and mixing angle, $m_{\tilde{t}_{1}}, m_{\tilde{t}_{2}}, \theta_{\tilde{t}}$, for large $\tan \beta$ also the scalar bottom masses and mixing angle, $m_{\tilde{b}_{1}}, m_{\tilde{b}_{2}}, \theta_{\hat{b}}$, the supersymmetric Higgs mass parameter, $\mu$, the U(1) and $\mathrm{SU}(2)$ gaugino masses, $M_{1}$ and $M_{2}$, and the gluino mass, $m_{\tilde{g}}$. These low-energy parameters are derived from the high-energy parameters of the three soft SUSY-breaking scenarios via RG running, see Ref. 32]. Since the RG running employed in the three scenarios is based on the $\overline{\mathrm{DR}}$ scheme, the corresponding low-energy parameters are $\overline{\mathrm{DR}}$ parameters. In order to derive predictions for observables, i.e. particle masses and mixing angles, these parameters in general have to be converted into on-shell parameters.

For the predictions in the MSSM Higgs sector we use results obtained in the Feynmandiagrammatic (FD) approach within the on-shell renormalization scheme as incorporated in the Fortran code FeynHiggs [38, 39, 40] based on Refs. [5, 4, 3].

Our analysis is concerned with the main Higgs production and decay channels at different colliders. To this end the predictions for the Higgs boson masses and effective couplings (especially the effective mixing angle, $\alpha_{\text {eff }}$, in the neutral $\mathcal{C} \mathcal{P}$-even Higgs boson sector that includes higher-order corrections) as well as for the branching ratios of the lightest MSSM Higgs boson (and for a SM Higgs boson with the same mass) have been evaluated. The effective mixing angle $\alpha_{\text {eff }}$ is defined via

$$
\alpha_{\mathrm{eff}}=\arctan \left[\frac{-\left(M_{A}^{2}+M_{Z}^{2}\right) \sin \beta \cos \beta-\hat{\Sigma}_{\phi_{1} \phi_{2}}}{M_{Z}^{2} \cos ^{2} \beta+M_{A}^{2} \sin ^{2} \beta-\hat{\Sigma}_{\phi_{1}}-m_{h}^{2}}\right], \quad-\frac{\pi}{2}<\alpha_{\mathrm{eff}}<\frac{\pi}{2} .
$$


Here $\hat{\Sigma}_{s}, s=\phi_{1}, \phi_{2}, \phi_{1} \phi_{2}$ denotes the renormalized Higgs boson self-energies in the $\phi_{1}-\phi_{2}$ basis. While the predictions for the decays of $h \rightarrow b \bar{b}, c \bar{c}, \tau^{+} \tau^{-}, \mu^{+} \mu^{-}$are based on Ref. [41, including the higher-order corrections described in Ref. 64, 65], the other decay channels have been derived with the code Hdecay [42, which has been implemented as a subroutine in the latest version of FeynHiggs [40]. The proper transition from on-shell parameters in FeynHiggs to $\overline{\mathrm{DR}}$ parameters in Hdecay has been taken into account [35, 36, 37.

In order to derive the relative difference between a MSSM production or decay rate and the corresponding SM rate (for the same Higgs boson mass), the following ratios have been calculated (below the notation $t \bar{t} \rightarrow t \bar{t} h$ refers to the processes $q \bar{q}, g g \rightarrow t \bar{t} h$, and $V V \rightarrow h$ refers to the vector boson fusion processes at the LHC and the LC, $q \bar{q} \rightarrow q^{\prime} \bar{q}^{\prime} h, e^{+} e^{-} \rightarrow \bar{\nu} \nu h$, respectively):

- $q \bar{q}, q q \rightarrow V \rightarrow V h(V=W, Z)$ :

$$
\frac{\sigma_{\mathrm{hV}}^{\mathrm{SUSY}}}{\sigma_{\mathrm{hV}}^{\mathrm{SM}}} \approx \sin ^{2}\left(\beta-\alpha_{\mathrm{eff}}\right)
$$

- $g g \rightarrow h:$

$$
\frac{\sigma^{\mathrm{SUSY}}(g g \rightarrow h)}{\sigma^{\mathrm{SM}}(g g \rightarrow h)} \approx \frac{\Gamma^{\mathrm{SUSY}}(h \rightarrow g g)}{\Gamma^{\mathrm{SM}}(h \rightarrow g g)}
$$

- $q \bar{q}, g g \rightarrow t \bar{t} \rightarrow t \bar{t} h:$

$$
\frac{\sigma^{\mathrm{SUSY}}(t \bar{t} \rightarrow t \bar{t} h)}{\sigma^{\mathrm{SM}}(t \bar{t} \rightarrow t \bar{t} h)} \approx \frac{\cos ^{2} \alpha_{\mathrm{eff}}}{\sin ^{2} \beta}
$$

- $V V \rightarrow h,(V=W, Z)$ :

$$
\frac{\sigma_{\mathrm{VVh}}^{\mathrm{SUSY}}}{\sigma_{\mathrm{VVh}}^{\mathrm{SM}}} \approx \sin ^{2}\left(\beta-\alpha_{\mathrm{eff}}\right)
$$

- $e^{+} e^{-} \rightarrow Z \rightarrow Z h$ :

$$
\frac{\sigma_{\mathrm{hZ}}^{\mathrm{SUSY}}}{\sigma_{\mathrm{hZ}}^{\mathrm{SM}}} \approx \sin ^{2}\left(\beta-\alpha_{\mathrm{eff}}\right)
$$

- $\gamma \gamma \rightarrow h$ :

$$
\frac{\sigma^{\mathrm{SUSY}}(\gamma \gamma \rightarrow h)}{\sigma^{\mathrm{SM}}(\gamma \gamma \rightarrow h)} \approx \frac{\Gamma^{\mathrm{SUSY}}(h \rightarrow \gamma \gamma)}{\Gamma^{\mathrm{SM}}(h \rightarrow \gamma \gamma)}
$$

- $\mu^{+} \mu^{-} \rightarrow h$ :

$$
\frac{\sigma^{\mathrm{SUSY}}\left(\mu^{+} \mu^{-} \rightarrow h\right)}{\sigma^{\mathrm{SM}}\left(\mu^{+} \mu^{-} \rightarrow h\right)} \approx \frac{\Gamma^{\mathrm{SUSY}}\left(h \rightarrow \mu^{+} \mu^{-}\right)}{\Gamma^{\mathrm{SM}}\left(h \rightarrow \mu^{+} \mu^{-}\right)}
$$


- Higgs boson decays:

$$
\begin{aligned}
& \frac{\mathrm{BR}^{\mathrm{SUSY}}(h \rightarrow b \bar{b})}{\mathrm{BR}^{\mathrm{SM}}(h \rightarrow b \bar{b})}, \frac{\mathrm{BR}^{\mathrm{SUSY}}(h \rightarrow c \bar{c})}{\mathrm{BR}^{\mathrm{SM}}(h \rightarrow c \bar{c})}, \frac{\mathrm{BR}^{\mathrm{SUSY}}\left(h \rightarrow \tau^{+} \tau^{-}\right)}{\mathrm{BR}^{\mathrm{SM}}\left(h \rightarrow \tau^{+} \tau^{-}\right)}, \\
& \frac{\mathrm{BR}^{\mathrm{SUSY}}(h \rightarrow \gamma \gamma)}{\mathrm{BR}^{\mathrm{SM}}(h \rightarrow \gamma \gamma)}, \frac{\mathrm{BR}^{\mathrm{SUSY}}\left(h \rightarrow W W^{*}\right)}{\mathrm{BR}^{\mathrm{SM}}\left(h \rightarrow W W^{*}\right)}, \frac{\mathrm{BR}^{\mathrm{SUSY}}(h \rightarrow g g)}{\mathrm{BR}^{\mathrm{SM}}(h \rightarrow g g)} .
\end{aligned}
$$

For some of the cross sections in Eqs. (8) - (13) more complete results for the MSSM exist in the literature than those used in our analysis, see e.g. Refs. 66, 67, 68, 69, For the qualitative analysis below, however, the approximations used here should be sufficient, see e.g. the comparison in 66.

For the numerical analysis the above production and decay modes have been combined to the most relevant channels for each collider, i.e. we have calculated the product

$$
\frac{\left.\sigma^{\mathrm{SUSY}} \text { (Higgs prod. }\right)}{\left.\sigma^{\mathrm{SM}} \text { (Higgs prod. }\right)} \times \frac{\left.\mathrm{BR}^{\mathrm{SUSY}} \text { (Higgs decay }\right)}{\left.\mathrm{BR}^{\mathrm{SM}} \text { (Higgs decay }\right)} \text {. }
$$

We take into account all possible Higgs boson decay channels including the full set of SUSY final states (in case the decay is kinematically allowed). This includes the invisible decay into the lightest neutralino, $h \rightarrow \tilde{\chi}_{1}^{0} \tilde{\chi}_{1}^{0}$. However, we have not found any region of parameters in the three soft SUSY-breaking scenarios in which this decay channel becomes sizable. In the following, we list the relevant Higgs boson production and decay channels for the various colliders:

- Tevatron:

$$
q \bar{q} \rightarrow V^{*} \rightarrow V h \rightarrow V b \bar{b},(V=W, Z)
$$

- LHC:

$$
\begin{aligned}
g g & \rightarrow h \rightarrow \gamma \gamma \\
q \bar{q}, g g \rightarrow t \bar{t} & \rightarrow t \bar{t} h \rightarrow t \bar{t} b \bar{b} \\
V V & \rightarrow h \rightarrow \tau^{+} \tau^{-}, W W^{*}
\end{aligned}
$$

- LC:

$$
\begin{aligned}
& e^{+} e^{-} \rightarrow Z^{*} \rightarrow Z h \rightarrow Z b \bar{b}, Z c \bar{c}, Z \tau^{+} \tau^{-}, Z W W^{*}, Z g g \\
& W W \rightarrow h \rightarrow b \bar{b}, c \bar{c}, \tau^{+} \tau^{-}, W W^{*}, g g
\end{aligned}
$$

Due to our approximations for the production processes the relative results of $\sigma^{\mathrm{SUSY}} / \sigma^{\mathrm{SM}} \times \mathrm{BR}^{\mathrm{SUSY}} / \mathrm{BR}^{\mathrm{SM}}$ for the same final states in the two chains numerically agree.

- $\gamma \mathrm{C}$ :

$$
\gamma \gamma \rightarrow h \rightarrow b \bar{b}, W W^{*}, \gamma \gamma
$$

- $\mu \mathrm{C}$ :

$$
\mu^{+} \mu^{-} \rightarrow h \rightarrow b \bar{b}, \tau^{+} \tau^{-}, W W^{*}
$$




\subsection{Phenomenological constraints}

While our main focus in this paper is on the physics in the Higgs sector, we also take into account some further (relatively mild) constraints when determining the allowed parameter space. These constraints are briefly summarized here. (A more detailed discussion can be found in Ref. [32.)

\section{- LEP Higgs bounds:}

The results from the Higgs search at LEP have excluded a considerable part of the MSSM parameter space [70]. The results of the search for the MSSM Higgs bosons are usually interpreted in three different benchmark scenarios [15]. The 95\% C.L. exclusion limit for the SM Higgs boson of $M_{H_{\mathrm{SM}}}>114.4 \mathrm{GeV}$ [2] applies also for the lightest $\mathcal{C P}$-even Higgs boson of the MSSM except for the parameter region with small $M_{A}$ and large $\tan \beta$. In the unconstrained MSSM this bound is reduced to $m_{h}>91.0 \mathrm{GeV}$ [70] for $M_{A} \lesssim 150 \mathrm{GeV}$ and $\tan \beta \gtrsim 8$ as a consequence of a reduced coupling of the Higgs to the $Z$ boson. For the $\mathcal{C P}$-odd Higgs boson a lower bound of $M_{A}>91.9 \mathrm{GeV}$ has been obtained [70]. In order to correctly interpolate between the parameter regions where the SM lower bound ${ }^{2}$ of $M_{H_{\mathrm{SM}}} \gtrsim 113 \mathrm{GeV}$ and the bound $m_{h} \gtrsim 91 \mathrm{GeV}$ apply, we use the result for the Higgs-mass exclusion given with respect to the reduced $Z Z h$ coupling squared (i.e. $\sin ^{2}\left(\beta-\alpha_{\text {eff }}\right)$ ) [71]. We have compared the excluded region with the theoretical prediction obtained at the two-loop level for $m_{h}$ and $\sin ^{2}\left(\beta-\alpha_{\text {eff }}\right)$ for each parameter set (using $m_{t}=175 \mathrm{GeV}$ ).

\section{- Precision observables:}

The electroweak precision observables are affected by the whole spectrum of SUSY particles. The main SUSY contributions enter via the $\rho$-parameter [72]. In our analysis we take into account the corrections arising from $\tilde{t} / \tilde{b}$ loops up to two-loop order [73]. A value of $\Delta \rho$ outside the experimentally preferred region of $\Delta \rho^{\mathrm{SUSY}} \lesssim 3 \times 10^{-3}$ [48] indicates experimentally disfavored $\tilde{t}$ and $\tilde{b}$ masses. The evaluation of $\Delta \rho^{\mathrm{SUSY}}$ is implemented in FeynHiggs.

\section{- Experimental bounds on SUSY particle masses}

In order to restrict the allowed parameter space in the three soft SUSY-breaking scenarios we employed the current experimental constraints on their low-energy mass spectrum [48. The precise values of the bounds that we have applied can be found in Ref. [32].

\section{- Other restrictions}

We just briefly list here the further restrictions that we have taken into account. For a detailed discussion see Ref. [32].

- The top quark mass is fixed to $m_{t}=175 \mathrm{GeV}$.

\footnotetext{
${ }^{2}$ Instead of the actual experimental lower bound, $M_{H_{\mathrm{SM}}} \gtrsim 114.4 \mathrm{GeV}$ [2], we use the value of $113 \mathrm{GeV}$ in order to allow for some uncertainty in the theoretical evaluation of $m_{h}$ from unknown higher-order corrections, which is currently estimated to be $\sim 3 \mathrm{GeV}$ [4].
} 
- The GUT or high-energy scale parameters are taken to be real, no SUSY $\mathcal{C} \mathcal{P}$ violating phases are assumed.

- In all models under consideration the $R$-parity symmetry [4] is taken to be conserved.

- Parameter sets that do not fulfil the condition ${ }^{3}$ of radiative electroweak symmetry breaking (REWSB) are discarded (already at the level of model generation).

- Parameter sets that do not fulfil the constraints that there should be no charge or color breaking minima are discarded (already at the level of model generation).

- Contrary to Ref. 32 we did not apply a "naturalness bound" on the sfermion and gluino mass, but just restricted the scanned parameter space as indicated in Sect. 2.1. This is in fact not an important restriction for the $\sigma \times$ BR calculation, since very heavy SUSY particles tend to decouple from the observables we are considering here, i.e. the quantity in Eq. (16) approaches 1.

- We demand that the lightest SUSY particle (LSP) is uncolored and uncharged. In the mGMSB scenario the LSP is always the gravitino, so this condition is automatically fulfilled. Within the mSUGRA and mAMSB scenario, the LSP is required to be the lightest neutralino. Parameter sets that result in a different LSP are excluded.

- We do not apply any further cosmological constraints, i.e. we do not demand a relic density in the region favored by dark matter constraints [75].

- Although we do not apply constraints from $\mathrm{BR}(b \rightarrow s \gamma)$ [50] or $g_{\mu}-2$ of the muon [49], we restrict ourselves to the case where the Higgsino mixing parameter is positive, $\mu>0$. This choice is favored by the current data. The results with negative $\mu$ can differ significantly from the case we consider here and would require an additional analysis.

\subsection{Bounds on $m_{h}$ and $\tan \beta$}

Scanning over the parameter space of mSUGRA, mGMSB and mAMSB as described in Sect. 2.1 and applying the constraints as described in Sect. 2.3 results in upper maximal values of $m_{h}$ and lower bounds on $\tan \beta$ (for the general MSSM case, see Refs. 70, 76 ). This analysis has been performed in Ref. 32. However, due to the progress in the $m_{h}$ evaluation, see Ref. 4] for a review, these bounds have changed as compared to our earlier analysis. Tab. 1 gives an update of the obtained $m_{h}$ and $\tan \beta$ bounds.

\section{Observability of the lightest MSSM Higgs boson}

In this section the observability of the lightest MSSM Higgs boson at the different colliders is analyzed. Especially at the hadron colliders a reduced $\sigma \times \mathrm{BR}$ for certain channels compared to the SM value could make it more difficult to establish a Higgs signal over the background.

\footnotetext{
${ }^{3}$ We use here the one-loop minimization conditions. Analytical two-loop expressions of $\mathcal{O}\left(\alpha_{t} \alpha_{s}+\alpha_{t}^{2}\right)$ for the minimization conditions have been recently given in Ref. [74]. The full two-loop corrections can be derived numerically from the work of Ref. [14].
} 


\begin{tabular}{|l||c|c|c|}
\cline { 2 - 4 } \multicolumn{1}{c||}{} & mSUGRA & mGMSB & mAMSB \\
\hline \hline$m_{h}^{\max }[\mathrm{GeV}]$ & 126.6 & 123.2 & 124.5 \\
\hline $\tan \beta^{\min }$ & 2.9 & 3.2 & 3.8 \\
\hline \hline
\end{tabular}

Table 1: Upper bound on $m_{h}, m_{h}<m_{h}^{\max }$, and lower bound on $\tan \beta, \tan \beta>\tan \beta^{\min }$, in the three soft SUSY-breaking scenarios (for $m_{t}=175 \mathrm{GeV}$ and taking into account the phenomenological constraints of Sect. 2.3] no theoretical uncertainties from unknown higherorder corrections are included in $m_{h}^{\max }$ ).

Before we discuss the observability of the lightest Higgs boson at the different colliders, we briefly summarize the main features of the Higgs boson couplings in dependence on the relevant SUSY parameters in the three soft SUSY-breaking scenarios. For a more extensive discussion see e.g. Ref. 32]. The most important parameters are $M_{A}$ and $\tan \beta$, since they enter the Higgs boson sector already at the tree-level. Deviations in the MSSM Higgs boson production and decay as compared to the SM prediction arise in particular from modifications in the $h \bar{b} b$ Yukawa coupling. The bottom and $\tau$ Yukawa coupling, being $\sim \sin \alpha_{\text {eff }} / \cos \beta$, can be strongly enhanced for small $M_{A}$ and large $\tan \beta$ values. This can lead to a strong enhancement of the partial widths $\Gamma(h \rightarrow b \bar{b}), \Gamma\left(h \rightarrow \tau^{+} \tau^{-}\right)$and therefore also of the total Higgs boson width. This gives rise to a corresponding suppression of the branching ratios of the other decay channels. In the case of mSUGRA, however, also a strong suppression of the $h \bar{b} b$ and $h \tau^{+} \tau$ - Yukawa couplings could happen for small $M_{A}$, large $\tan \beta$ and large $\mu$ due to radiative corrections leading to a small value of $\sin \alpha_{\mathrm{eff}}$.

- Tevatron:

In all three soft SUSY-breaking scenarios the Tevatron search channels are not significantly suppressed compared to the SM rates. The channel $V \rightarrow V h \rightarrow V b \bar{b}$ is suppressed by not more than $10 \%$ as compared to the SM value. Therefore the prospects at the Tevatron for the discovery of the lightest MSSM Higgs boson are as good as for the SM Higgs boson. A similar observation has already been made in Ref. [43] for the mSUGRA scenario, where also other phenomenological constraints have been taken into account.

- LHC:

We start our discussion with the channel $g g \rightarrow h \rightarrow \gamma \gamma$ that is of particular importance for a light SM-like Higgs boson with $m_{h} \lesssim 130 \mathrm{GeV}$ [77]. As has been shown in Ref. [78, 79, 16], for certain values of the SUSY parameters this production channel can be heavily suppressed for a wide region of the $M_{A}-\tan \beta$-parameter space of the unconstrained MSSM (see also Ref. [80] for an analysis in the mSUGRA scenario). Since the event rate for this channel is relatively low ( $h \rightarrow \gamma \gamma$ being a rare decay), a suppression of $50 \%$ or more would certainly pose a challenge to the experiment.

The situation within the three soft SUSY-breaking scenarios can be read off from Fig. $\mathbb{1}^{4}$. Within the mSUGRA scenario a suppression of up to $10-20 \%$ is found for $M_{A} \lesssim 700 \mathrm{GeV}$ for all $\tan \beta$ values. For very large $\tan \beta, \tan \beta \gtrsim 50$, a reduction even

\footnotetext{
${ }^{4}$ For a similar analysis for the charged Higgs bosons, see Ref. 81].
} 

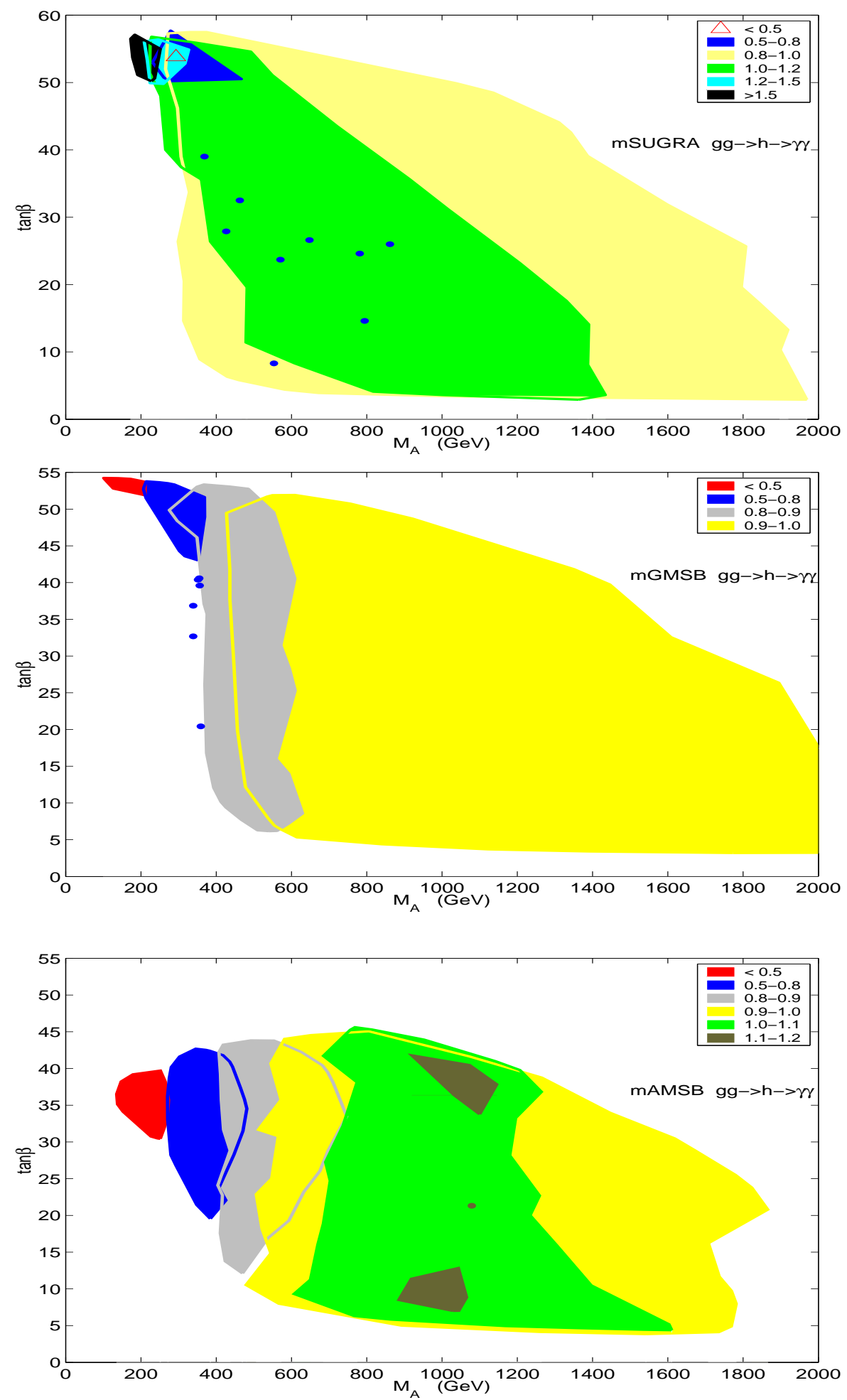

Figure 1: The rate for the LHC channel $g g \rightarrow h \rightarrow \gamma \gamma$, normalized to the SM case with the same Higgs boson mass, $(\sigma \times \mathrm{BR})^{\mathrm{SUSY}} /(\sigma \times \mathrm{BR})^{\mathrm{SM}}$, is shown in the $M_{A}-\tan \beta$ plane for the mSUGRA, mGMSB and mAMSB scenarios (from top to bottom). The unconnected dots appearing in this and the following figures reflect the fact that only a few points with very low density appear in this area. However, increasing the density of the scatter data would cover the whole area in which the dots are located. 
larger than $20 \%$ can be found. This suppression is due to the fact that the $\mathrm{BR}(h \rightarrow b \bar{b})$ is strongly enhanced in this part of the parameter space, as explained in the beginning of this section. This is in agreement with the result obtained in Ref. 43, where no substantial reduction has been found, after other phenomenological constraints like $\mathrm{BR}(b \rightarrow s \gamma), g_{\mu}-2$ and especially the CDM restrictions had been applied. Concerning the parameter space of the GUT scale parameters $m_{0}$ and $m_{1 / 2}$ (which is not shown here explicitly), the reduction is found for all $m_{0}$ and $m_{1 / 2} \lesssim 350 \mathrm{GeV}$. It should be noticed that an enhancement of $50 \%$ or more is possible in the very small $M_{A}$ region due to a possible strong suppression of $h \rightarrow b \bar{b}$ in accordance with the analysis in Ref. 32 .

The suppression can be stronger in the other two scenarios. Nearly all model points with $M_{A} \lesssim 400 \mathrm{GeV}$ for mGMSB and mAMSB, which correspond to $\tan \beta$ values of $\tan \beta \gtrsim 20$, show a suppression of $20-50 \%$ (or even more). For $M_{A} \lesssim 200 \mathrm{GeV}(300 \mathrm{GeV}$ ) and $\tan \beta \gtrsim 50(30 \lesssim \tan \beta \lesssim 40)$ the reduction is even larger than $50 \%$ in mGMSB (mAMSB). For larger $M_{A}$ values, $M_{A} \gtrsim 600 \mathrm{GeV}$, the $\mathrm{SM}$ value of $\sigma \times \mathrm{BR}$ is approached. Concerning the high-energy parameters, the largest reduction in the mGMSB scenario is found all over the parameter space for $M_{\text {mess }}$ and $N_{\text {mess }}$ (with $\tan \beta \gtrsim 50$ and $\Lambda \gtrsim 30 \mathrm{TeV}$ ), whereas a reduction by $10-20 \%$ is mostly found for the lowest $\Lambda$ values for all $M_{\text {mess }}$ values. Within mAMSB the largest reduction is found for $m_{0} \lesssim 700 \mathrm{GeV}$ and $m_{\text {aux }} \lesssim 4 \times 10^{4} \mathrm{GeV}$. Small values of the high-energy parameters correspond to relatively small values of the low-energy SUSY parameters, which are required for a sizable suppression of the $g g \rightarrow h \rightarrow \gamma \gamma$ channel [78, 79, 16]. The fact that the suppression of this channel can be much more pronounced in mGMSB and mAMSB as compared to mSUGRA originates to a large extent from the different behavior of the $\mathrm{BR}(h \rightarrow b \bar{b})$ channel (see also below). This dominant decay channel can be much more strongly enhanced in mGMSB and mABSB [32] and thus suppress the decay of the lightest Higgs boson to photons.

It is interesting to note that only in the mAMSB scenario an enhancement of the $g g \rightarrow h \rightarrow \gamma \gamma$ can be found in the intermediate $M_{A}$ region, which is absent in the other scenarios (within mSUGRA the region of enhancement labeled with "1.0 - 1.2" in general has values very close to 1.0). The reason for this enhancement is the following combination of effects: for the intermediate $M_{A}$ region, the partial decay width $\Gamma(h \rightarrow$ $\gamma \gamma$ ) can be slightly enhanced due to loop corrections in mSUGRA and mGMSB (less then 10\%), while it is more enhanced in mAMSB (up to 20\%). The total decay width is dominated by $\Gamma(h \rightarrow b \bar{b})$, which can be enhanced up to $10-20 \%$ in mGMSB, but $10 \%$ at most within the mAMSB scenario. This results in a suppression (or at most only a very mild increase) of $\mathrm{BR}(h \rightarrow \gamma \gamma)$ within mSUGRA and mGMSB as compared to the SM, but in an enhancement for mAMSB in the intermediate $M_{A}$ region.

We now consider the associated production channel at the LHC, $q \bar{q}, g g \rightarrow t \bar{t} \rightarrow t \bar{t} h \rightarrow$ $t \bar{t} b \bar{b} .{ }^{5}$ In all three scenarios the rate is not suppressed compared to the SM rate by more than $10 \%$ or even an enhancement by up to $10-20 \%$ occurs. Therefore this channel, which is most relevant in the region $100 \mathrm{GeV} \lesssim m_{h} \lesssim 120 \mathrm{GeV}$ [77], does not suffer

${ }^{5}$ Also some other channels for a SM Higgs boson have been studied, e.g. $t \bar{t} \rightarrow t \bar{t} h \rightarrow t \bar{t} W W^{*}$ 82. They could be easily included in our analysis as outlined in Sect. 22 Since including these channels does not change our qualitative results, we do not discuss them here explicitly. 
from suppression due to SUSY corrections in the three soft SUSY-breaking scenarios. More severe suppressions are possible for the $q \bar{q}, g g \rightarrow b \bar{b} h$ channel. This channel can be observed for very small $M_{A}$ and large $\tan \beta$, but plays only a minor role concerning the observability of the lightest MSSM Higgs boson at the LHC 83].

More recently also the Higgs boson production via $W$ boson fusion, $W^{+} W^{-} \rightarrow h$, with a subsequent decay to $\tau^{+} \tau^{-}$pairs, $W$ bosons or photons has been discussed, see Refs. 84, 85, for a SM analyses and Refs. 86, 87, for the mode $h \rightarrow \tau^{+} \tau^{-}$in the MSSM case. These channels can be relevant for the whole allowed $m_{h}$ range in the MSSM. The mSUGRA scenario offers very good prospects for the decay into $\tau^{+} \tau^{-}$, which is characterized by the coupling $\sim \sin \alpha_{\text {eff }} / \cos \beta$. Over almost the whole parameter space the rate for $\sigma \times \mathrm{BR}$ differs by less than $10 \%$ from the SM rate. Only for very small $M_{A}$ and very high $\tan \beta$ a suppression is possible. This corresponds to the parameter space where the heavy Higgs boson can have SM like couplings, see Fig. 1 in Ref. [32. The other channels $\left(h \rightarrow W W^{*}, \gamma \gamma\right.$ ) show the following pattern: due to the increased decay rates to fermions, at relatively small $M_{A}, 250 \mathrm{GeV} \lesssim M_{A} \lesssim 500 \mathrm{GeV}$, a suppression of the decays into $W$ bosons or photons is possible, while the value of the SM rate is approached for larger $M_{A}$.

The situation is similar in the mGMSB and mAMSB scenarios, besides that the suppression of the $\tau^{+} \tau^{-}$channel for very small $M_{A}$ and very large $\tan \beta$ is not found. For the smallest possible values of $M_{A}$ and the largest possible values of $\tan \beta$ a strong enhancement of these channels can be observed. This agrees with the results of Ref. [32, where only in the mSUGRA scenario but not in the mGMSB and mAMSB scenario a region of the parameter space has been found where the heavy Higgs boson is SM like. Thus the $h \rightarrow \tau^{+} \tau^{-}$channel is enhanced everywhere in mGMSB and mAMSB. Correspondingly the two other channels, $h \rightarrow W W^{*}$ and $h \rightarrow \gamma \gamma$ are suppressed everywhere. As an example, in Fig. 2] we show the channel $W W \rightarrow h \rightarrow W W^{*}$ in the mGMSB and the mAMSB scenario. For not too large $M_{A}, M_{A} \lesssim 550 \mathrm{GeV}(700 \mathrm{GeV})$, in mGMSB (mAMSB) a reduction larger than $10 \%$ can be observed. For larger $M_{A}$ the results in the mGMSB and mAMSB scenarios approach the one in the SM.

- LC:

Due to its clean experimental environment, Higgs boson production should be easily observable at the LC, i.e. in various channels even a significant suppression compared to the SM rate would not be harmful [88, 89, 90]. Therefore the production channels $Z^{*} \rightarrow Z h$ and $W W \rightarrow h$ (which yield the same numerical result in our analysis, see Sect. 2.2) and all decay channels, $h \rightarrow b \bar{b}, c \bar{c}, \tau^{+} \tau^{-}, W W^{*}, g g$, are observable in the three soft SUSY-breaking scenarios. This applies also in the region of small $M_{A}$, $M_{A} \lesssim 200 \mathrm{GeV}$, where a suppression of more than $50 \%$ can occur in all three scenarios. We will therefore present a detailed analysis of the LC production and decay channels only in the context of precision measurements, see Sect. 4.

- $\gamma \mathrm{C}$ :

Also the $\gamma \mathrm{C}$, due to the Higgs boson production in the s-channel, offers very good prospects for the Higgs boson observation [91, 44. Only the decay $h \rightarrow \gamma \gamma$ could 

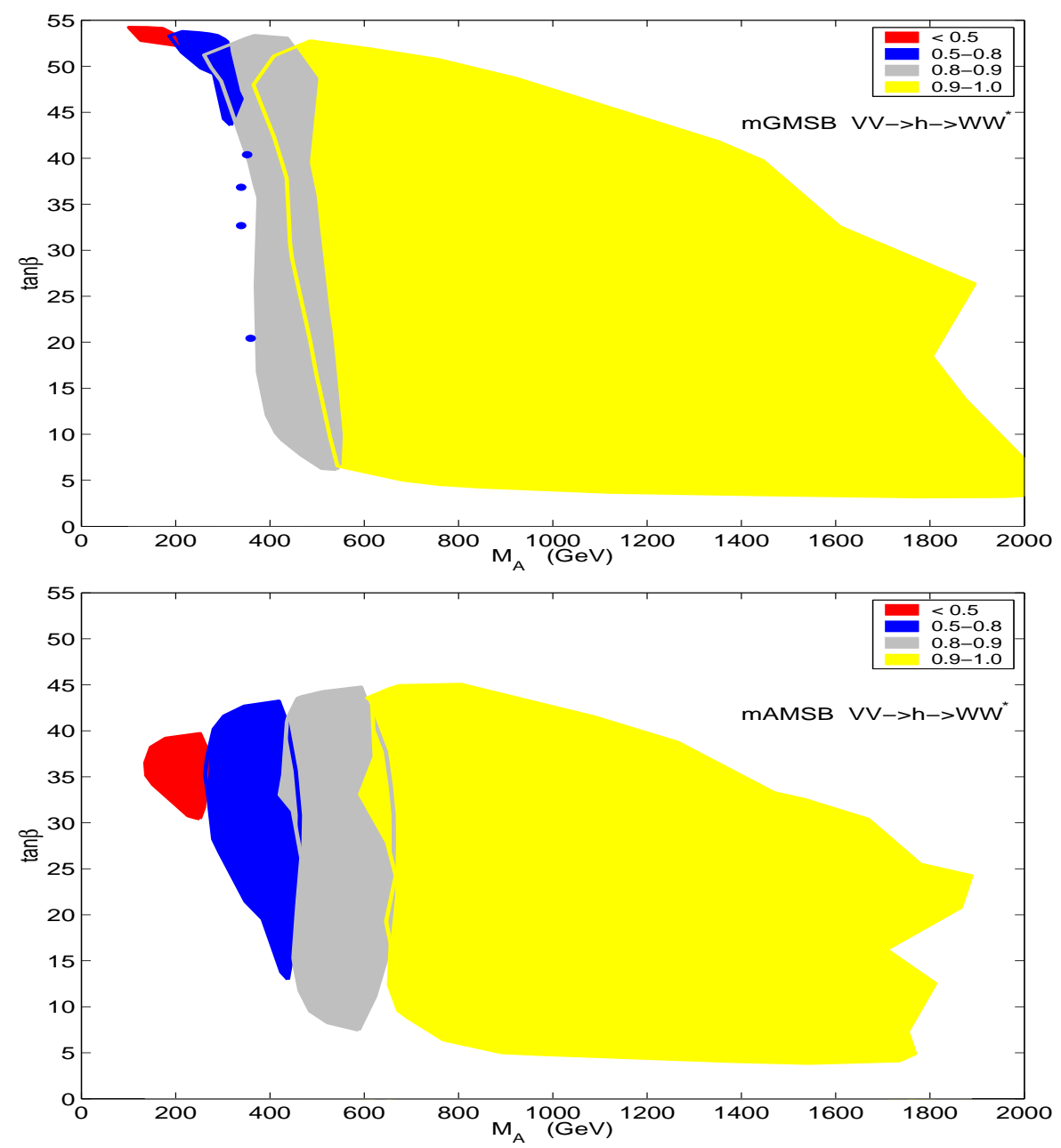

Figure 2: The rate for the LHC channel $W W \rightarrow h \rightarrow W W^{*}$, normalized to the SM case with the same Higgs boson mass, is shown in the $M_{A}-\tan \beta$ plane for the mGMSB (upper plot) and the mAMSB scenario (lower plot).

become problematic if it is strongly suppressed compared to the SM value (e.g. due to an enhanced $h b \bar{b}$ coupling).

In the mSUGRA scenario the $h \rightarrow \gamma \gamma$ channel and the $h \rightarrow W W^{*}$ channel are very similar. They can be suppressed by more than $20 \%$ only for very large $\tan \beta, \tan \beta \gtrsim 50$, or for $\tan \beta \approx 10$ with the smallest allowed $M_{A}$ values. On the other hand, the $h \rightarrow b \bar{b}$ and $h \rightarrow \tau^{+} \tau^{-}$modes are unproblematic within mSUGRA and show either only a very small suppression or even some enhancement, with the only exception of a possible suppression at very large $\tan \beta$.

In the mGMSB scenario the $h \rightarrow b \bar{b}$ and $h \rightarrow \tau^{+} \tau^{-}$channels are always enhanced. The $h \rightarrow \gamma \gamma$ and $h \rightarrow W W^{*}$ channels show a suppression of more than $50 \%$ for $M_{A} \lesssim 100 \mathrm{GeV}$, while for $M_{A} \lesssim 600 \mathrm{GeV}$ still a suppression of more than $10 \%$ occurs, see Fig. 3 for the $h \rightarrow \gamma \gamma$ channel. The situation is quite similar in the mAMSB scenario, with an exception in the intermediate $M_{A}$ region, $600-1300 \mathrm{GeV}$, where 

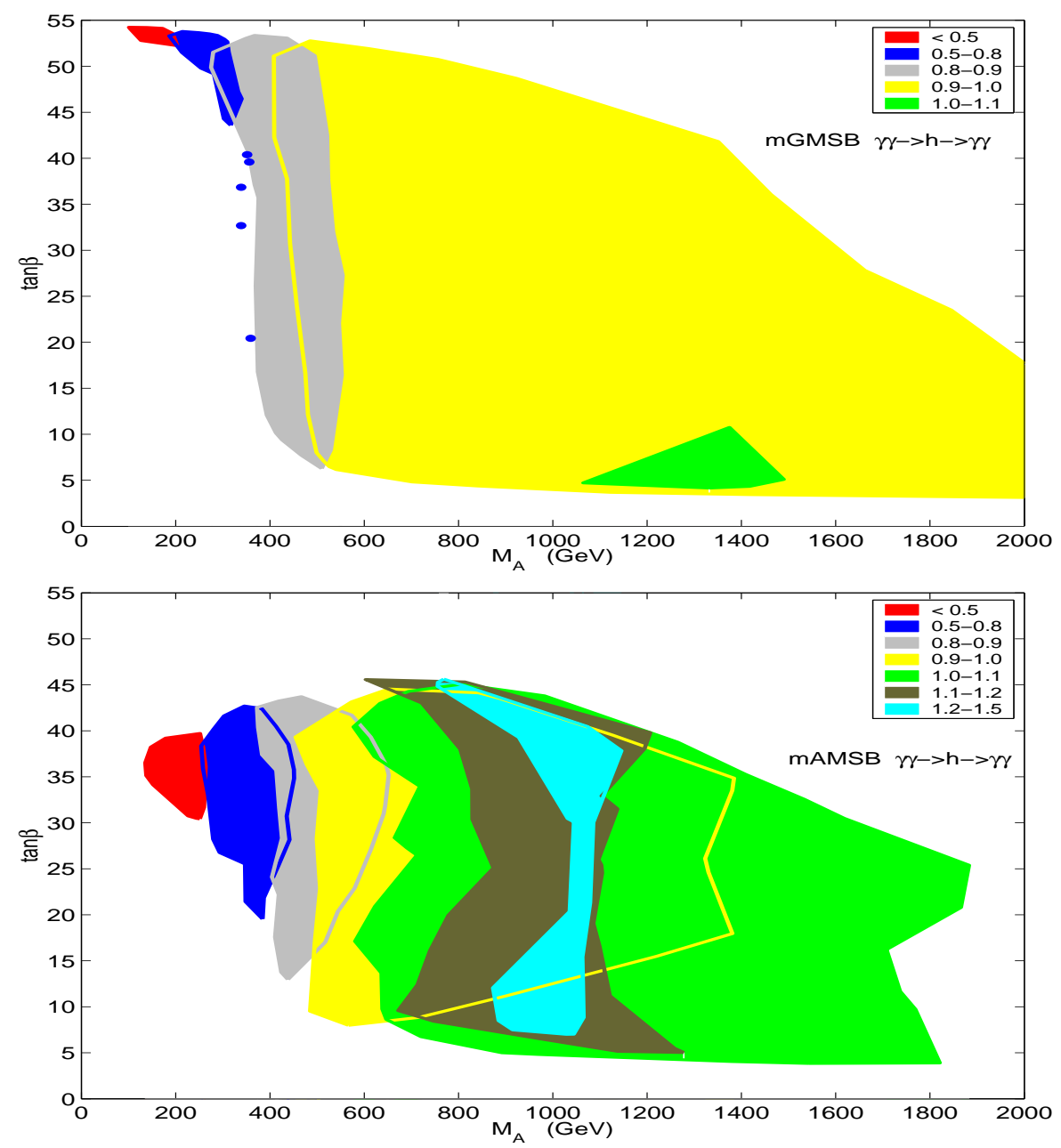

Figure 3: The rate for the $\gamma \mathrm{C}$ channel $\gamma \gamma \rightarrow h \rightarrow \gamma \gamma$, normalized to the SM case with the same Higgs boson mass, is shown in the $M_{A}-\tan \beta$ plane for the mGMSB (upper plot) and the mAMSB scenario (lower plot).

there is an enhancement of the $h \rightarrow \gamma \gamma$ mode compared to the one obtained in the mGMSB scenario, see Fig. 3. The reason for this enhancement is similar to the case of the $g g \rightarrow h \rightarrow \gamma \gamma$ channel at the LHC (see also the discussion of Fig. 1). There the branching ratio for $h \rightarrow \gamma \gamma$ is enhanced in mAMSB as compared to mGMSB (and mSUGRA). At the $\gamma \mathrm{C}$ the effect is even more pronounced since the enhanced $\gamma \gamma h$ vertex now also enters in the Higgs production. Correspondingly, the $\gamma \gamma \rightarrow h \rightarrow \gamma \gamma$ channel can be only slightly enhanced in mGMSB, but more strongly increased in the mAMSB scenario. For the process $V V \rightarrow h \rightarrow W W^{*}$ that is important at the LHC and the LC, see e.g. Fig. 2, the mGMSB and the mAMSB scenario are very similar, since no $h \gamma \gamma$ vertex is involved.

- $\mu \mathrm{C}$ :

Finally, to complete our analysis, we also briefly look at the $\mu \mathrm{C}$. This collider offers good prospects since the Higgs boson can be produced in the s-channel without a 
loop suppression like at the $\gamma \mathrm{C}$, however with the relatively small $\mu^{+} \mu^{-} h$ Yukawa coupling [92,93]. The production of SUSY Higgs bosons at the $\mu \mathrm{C}$ has been extensively discussed in the literature, see e.g. Refs. [92,93] and references therein, but the impact of the different SUSY-breaking scenarios has not been investigated yet. In the unconstrained MSSM it is possible that the $\mu^{+} \mu^{-} h$ coupling, being $\sim \sin \alpha_{\text {eff }} / \cos \beta$, can become very small if $\alpha_{\text {eff }} \rightarrow 0$ because of loop corrections [93, 94. In this parameter region, on the other hand, $H, A$ production at the $\mu \mathrm{C}$ happens with an enhanced rate and offers good prospects for resolving $H$ and $A$ as separate resonances [93, 94].

The feature of a suppressed $\mu^{+} \mu^{-} h$ coupling can also be realized in the mSUGRA scenario when the heavy (and not the light) $\mathcal{C P}$-even Higgs boson is SM like. This is possible for very high $\tan \beta$ and small $M_{A}, M_{A} \lesssim 300 \mathrm{GeV}$ (note that here no CDM constraints are taken into account, in contrast to the analysis of Ref. [93]). In this parameter region a strong suppression is possible for all $\mu \mathrm{C}$ channels of the light $\mathcal{C P}$-even Higgs boson (while $H, A$ production happens at enhanced rates). In the rest of the parameter space the $h \rightarrow b \bar{b}$ and $h \rightarrow \tau^{+} \tau^{-}$channel are strongly enhanced for $M_{A} \lesssim 700 \mathrm{GeV}$. For very large $M_{A}$ an enhancement of up to $10 \%$ occurs. Correspondingly, the $h \rightarrow W W^{*}$ channel is not enhanced, but still within $10 \%$ of the SM value.

Within mGMSB and mAMSB the suppression of the $\mu^{+} \mu^{-} h$ coupling is not present. Because of the coupling factor $\sin \alpha_{\text {eff }} / \cos \beta$ the $h \rightarrow b \bar{b}$ and $h \rightarrow \tau^{+} \tau^{-}$channels are strongly enhanced for small $M_{A}$, while the SM value is approached for large values of $M_{A}$. The $h \rightarrow W W^{*}$ channel, being enhanced with the $\sin \alpha_{\text {eff }} / \cos \beta$ factor only at the production vertex, is less enhanced, but should be unproblematic for the whole parameter space.

The results of this section are summarized in Tab. 2. The modes $g g \rightarrow h \rightarrow \gamma \gamma, t \bar{t} \rightarrow t \bar{t} h$ and $W W \rightarrow h \rightarrow W W^{*}, \tau^{+} \tau^{-}, \gamma \gamma$ allow the detection of the lightest MSSM Higgs boson in all three scenarios over the whole indicated parameter space. Possible exceptions occur for the $g g \rightarrow h \rightarrow \gamma \gamma$ channel for the very small $M_{A}$ region in the mGMSB and mAMSB scenarios, where a strong suppression of more than $50 \%$ could happen. Because of the clean experimental environment at a LC, detection of the light Higgs is ensured for all the three scenarios. For a $\gamma \mathrm{C}$, the region of very small $M_{A}$ values in the mGMSB and mAMSB scenarios might be difficult for the $h \rightarrow W W^{*}$ and $h \rightarrow \gamma \gamma$ mode because of a strong suppression of more than $50 \%$. At the $\mu \mathrm{C}$, the region $M_{A} \lesssim 150 \mathrm{GeV}$ and $\tan \beta \gtrsim 50$ in the mSUGRA scenario exhibits a significant suppression of the production of the lightest $\mathcal{C} \mathcal{P}$-even MSSM Higgs boson. Besides the "difficult" regions mentioned above, the main search modes at the $\gamma \mathrm{C}$ and the $\mu \mathrm{C}$ are not affected by strong suppression for all three scenarios, and the lightest MSSM Higgs boson in these scenarios will clearly be detectable at all possible future colliders.

\section{Precision analyses of the Higgs masses and branch- ing ratios}

We now investigate the potential of Higgs branching ratio measurements at future colliders for testing the underlying SUSY model. We concentrate our analysis on the LC and the 


\begin{tabular}{|c|c|c|c|c|c|}
\hline Channel & Collider & $m_{h}$ & mSUGRA & mGMSB & mAMSB \\
\hline$V^{*} \rightarrow V h \rightarrow V b \bar{b}$ & $\begin{array}{l}\text { Tev } \\
\text { LC }\end{array}$ & $\begin{array}{l}\lesssim 135 \\
\lesssim 0.7 \sqrt{s}\end{array}$ & $\pm 10 \%$ & $\pm 10 \%$ & $M_{A} \lesssim 300:+50 \%$ \\
\hline$V^{*} \rightarrow V h \rightarrow V c \bar{c}$ & $\mathrm{LC}$ & $\lesssim 0.7 \sqrt{s}$ & $M_{A} \lesssim 500: \pm 20 \%$ & $\begin{aligned} & 400 \lesssim M_{A} \lesssim 600:-20 \% \\
& 200 \lesssim M_{A} \lesssim 400:-50 \% \\
& M_{A} \lesssim 200: \lesssim-50 \%\end{aligned}$ & $\begin{aligned} & 500 \lesssim M_{A} \lesssim 650:-20 \% \\
& 300 \lesssim M_{A} \lesssim 500:-50 \% \\
& M_{A} \lesssim 300: \lesssim-50 \%\end{aligned}$ \\
\hline $\begin{array}{c}V V \rightarrow h \rightarrow W W^{*} \\
V^{*} \rightarrow V h \rightarrow V W W^{*}\end{array}$ & $\begin{array}{c}\mathrm{LHC} \\
\mathrm{LC}\end{array}$ & $\begin{array}{l}\gtrsim 110 \\
\lesssim 0.7 \sqrt{s}\end{array}$ & $\begin{array}{c}M_{A} \lesssim 150: \pm 20 \% \\
\text { for } \tan \beta \lesssim 30 \text { or } \gtrsim 50\end{array}$ & like $V c \bar{c}$ & like $V c \bar{c}$ \\
\hline $\begin{array}{c}V V \rightarrow h \rightarrow \tau^{+} \tau^{-} \\
V^{*} \rightarrow V h \rightarrow V \tau^{+} \tau^{-}\end{array}$ & $\begin{array}{c}\mathrm{LHC} \\
\mathrm{LC}\end{array}$ & $\begin{array}{l}\lesssim 150 \\
\lesssim 0.7 \sqrt{s}\end{array}$ & $\begin{array}{c}M_{A} \lesssim 150: \pm 50 \% \\
\text { for } \tan \beta \gtrsim 50\end{array}$ & $\tan \beta \gtrsim 50: \gtrsim 50 \%$ & $M_{A} \lesssim 500:+50 \%$ \\
\hline$V^{*} \rightarrow V h \rightarrow V g g$ & $\mathrm{LC}$ & $\lesssim 130$ & $M_{A} \lesssim 200: \pm 20 \%$ & like $V c \bar{c}$ & $\begin{aligned} & 550 \lesssim M_{A} \lesssim 650:-20 \% \\
& 250 \lesssim M_{A} \lesssim 550:-50 \% \\
& M_{A} \lesssim 250: \lesssim-50 \%\end{aligned}$ \\
\hline$g g \rightarrow h \rightarrow \gamma \gamma$ & LHC & $\lesssim 150$ & $\begin{array}{l}M_{A} \lesssim 150: \pm 20 \% \\
\tan \beta \lesssim 30 \text { or } \gtrsim 50\end{array}$ & like $V c \bar{c}$ & $\begin{aligned} & 550 \lesssim M_{A} \lesssim 750:-20 \% \\
& 250 \lesssim M_{A} \lesssim 550:-50 \% \\
& M_{A} \lesssim 250: \lesssim-50 \%\end{aligned}$ \\
\hline$t \bar{t} \rightarrow t \bar{t} h \rightarrow t \bar{t} b \bar{b}$ & LHC & $\lesssim 120$ & SM like & $\begin{array}{c}\text { SM like } \\
+50 \% \text { for } \tan \beta \gtrsim 50\end{array}$ & $M_{A} \lesssim 300:+50 \%$ \\
\hline$\gamma \gamma \rightarrow h \rightarrow b \bar{b}$ & $\gamma \mathrm{C}$ & n.f.e. & $M_{A} \lesssim 150:+50 \%$ & SM like & $M_{A} \lesssim 300:+50 \%$ \\
\hline$\gamma \gamma \rightarrow h \rightarrow W W^{*}$ & $\gamma \mathrm{C}$ & n.f.e. & $\begin{array}{c}M_{A} \lesssim 200: \\
-20 \% \text { up to }+50 \%\end{array}$ & $\begin{aligned} & 400 \lesssim M_{A} \lesssim 600:-20 \% \\
& 200 \lesssim M_{A} \lesssim 400:-50 \% \\
& M_{A} \lesssim 200: \lesssim-50 \%\end{aligned}$ & $\begin{aligned} 500 & \lesssim M_{A} \lesssim 650:-20 \% \\
250 & \lesssim M_{A} \lesssim 500:-50 \% \\
M_{A} & \lesssim 250: \lesssim-50 \%\end{aligned}$ \\
\hline$\gamma \gamma \rightarrow h \rightarrow \tau^{+} \tau^{-}$ & $\gamma \mathrm{C}$ & n.f.e. & $\begin{array}{c}\tan \beta \sim 50: \pm 50 \% \\
\tan \beta<50:+50 \% \\
\quad \text { for } M_{A} \lesssim 150\end{array}$ & $\tan \beta \gtrsim 50: \gtrsim 50 \%$ & $M_{A} \lesssim 600:+50 \%$ \\
\hline$\gamma \gamma \rightarrow h \rightarrow \gamma \gamma$ & $\gamma \mathrm{C}$ & n.f.e. & $\begin{array}{c}M_{A} \lesssim 200: \\
-20 \% \text { up to }+50 \%\end{array}$ & like $\gamma \gamma \rightarrow h \rightarrow W W^{*}$ & $\begin{aligned} & 500 \lesssim M_{A} \lesssim 650:-20 \% \\
& 250 \lesssim M_{A} \lesssim 500:-50 \% \\
& M_{A} \lesssim 250: \lesssim-50 \%\end{aligned}$ \\
\hline$\mu^{+} \mu^{-} \rightarrow h \rightarrow b \bar{b}$ & $\mu \mathrm{C}$ & n.f.e. & $\begin{array}{c}M_{A} \lesssim 250:+50 \% \\
M_{A} \lesssim 150, \tan \beta>50:-50 \%\end{array}$ & $\begin{array}{c}200 \lesssim M_{A} \lesssim 600:+50 \% \\
M_{A} \lesssim 200: \gtrsim 50 \%\end{array}$ & $\begin{array}{c}450 \lesssim M_{A} \lesssim 900:+50 \% \\
M_{A} \lesssim 450: \gtrsim 50 \%\end{array}$ \\
\hline$\mu^{+} \mu^{-} \rightarrow h \rightarrow W W^{*}$ & $\mu \mathrm{C}$ & n.f.e. & $\pm 10 \%$ & $\tan \beta \simeq 50:+50 \%$ & $M_{A} \lesssim 600:+50 \%$ \\
\hline$\mu^{+} \mu^{-} \rightarrow h \rightarrow \tau^{+} \tau^{-}$ & $\mu \mathrm{C}$ & n.f.e. & $\begin{array}{c}M_{A} \lesssim 250:+50 \% \\
M_{A} \lesssim 150, \tan \beta>50:-50 \%\end{array}$ & like $\mu^{+} \mu^{-} \rightarrow h \rightarrow b \bar{b}$ & like $\mu^{+} \mu^{-} \rightarrow h \rightarrow b \bar{b}$ \\
\hline
\end{tabular}

Table 2: Behavior of the production and decay modes of the lightest $\mathcal{C} \mathcal{P}$-even Higgs boson of the MSSM, see Eq. (16), for the most relevant channels at present and future colliders in three different SUSY breaking scenarios. When there is suppression or enhancement we indicate its maximum magnitude together with the parameters where this happens. If not stated explicitly, for the rest of the parameter space the mode behaves roughly like in the SM. The Higgs mass range where detection of a statistically significant signal is possible is also shown [95,96]. The phrase "n.f.e." stands for "not fully explored" and refers to channels where the studies so far have been performed for some fixed $m_{h}$ values only. 
$\gamma \mathrm{C}$, since the anticipated precisions of at the LHC will in general be much worse, while on the other hand branching ratio measurements of the light Higgs boson at the $\mu \mathrm{C}$ are not expected to yield substantially better results than at the LC.

Over most of the parameter space of the scenarios discussed here one would of course also expect to observe direct production of SUSY particles at the next generation of colliders. However, we concentrate our analysis on information obtainable from the Higgs sector without assuming further knowledge of the SUSY spectrum. In a realistic situation one would of course confront the model under study with all available experimental information.

Tab. 3 lists the anticipated accuracies in different channels at the LC [88, 97] and the $\gamma \mathrm{C}$ [91,44]. The values given in Tab. 3 correspond to a SM-like Higgs boson with a mass compatible with the allowed mass range of the lightest $\mathcal{C} \mathcal{P}$-even Higgs boson in the three soft SUSY-breaking scenarios according to the upper bounds given in Tab. 11. There is of course some variation in the accuracy with which the branching ratios can be measured over the allowed range of $m_{h}$. For simplicity, we assume a constant precision over the allowed mass range for each channel with a value referring to the middle of the allowed range. In parameter regions where the MSSM rate differs drastically from the SM rate the prospective precision will of course be different than in the SM. While in extreme cases like this it will be easy to infer properties of the SUSY model from Higgs sector measurements, we will focus in our analysis below on moderate deviations between the MSSM and the SM, for which the values given in Tab. 3 can be applied in good approximation. We will indicate the deviation between the MSSM and the SM in terms of the accuracies given in Tab. 3, i.e. a " $\pm n \sigma$ " deviation means that the calculated MSSM value of $\sigma \times \mathrm{BR}$ deviates from the corresponding SM value (with $\left.M_{H_{\mathrm{SM}}}=m_{h}\right)$ by $( \pm n \times$ precision $)$.

\begin{tabular}{|c|l|c|}
\hline \hline collider & decay mode & precision \\
\hline \hline $\mathrm{LC}$ & $h \rightarrow b \bar{b}$ & $1.5 \%$ \\
\hline $\mathrm{LC}$ & $h \rightarrow \tau^{+} \tau^{-}$ & $4.5 \%$ \\
\hline $\mathrm{LC}$ & $h \rightarrow c \bar{c}$ & $6 \%$ \\
\hline $\mathrm{LC}$ & $h \rightarrow g g$ & $4 \%$ \\
\hline $\mathrm{LC}$ & $h \rightarrow W W^{*}$ & $3 \%$ \\
\hline \hline$\gamma \mathrm{C}$ & $h \rightarrow b \bar{b}$ & $2 \%$ \\
\hline$\gamma \mathrm{C}$ & $h \rightarrow W W^{*}$ & $5 \%$ \\
\hline$\gamma \mathrm{C}$ & $h \rightarrow \gamma \gamma$ & $11 \%$ \\
\hline \hline
\end{tabular}

Table 3: Anticipated precisions for measurements of Higgs branching ratios at the LC [88, 97 ] and the $\gamma \mathrm{C}$ [91,44]. The values are given for a SM-like Higgs boson with a mass compatible with the allowed mass range of the lightest $\mathcal{C} \mathcal{P}$-even Higgs boson in the three soft SUSYbreaking scenarios, see text. 


\subsection{Sensitivity to $M_{A}$ and $\tan \beta$}

While within the MSSM the prospects for the detection of the lightest $\mathcal{C P}$-even Higgs boson at the next generation of colliders are very good, the situation is quite different for direct observation of the $\mathcal{C P}$-odd $A$ boson. At the LHC the detection of this particle can be very difficult over sizable fractions of the MSSM parameter space (see e.g. Ref. [77, 98]), while it may be outside the kinematical reach of the LC (see Refs. [67, 99] for a recent account of this subject). Thus, it is of interest to study the potential for obtaining indirect bounds on $M_{A}$ from precision measurements. Exploiting the sensitivity to $M_{A}$ can be done in a similar fashion as nowadays for the SM Higgs, where indirect bounds are derived from electroweak precision tests. Since in the decoupling limit, $M_{A} \gg M_{Z}$, the Higgs sector of the MSSM becomes SM-like, deviations in the production and decay of the lightest $\mathcal{C} \mathcal{P}$-even Higgs boson of the MSSM can in principle be translated into an upper bound on $M_{A}$. If direct information on $M_{A}$ is available, the indirect sensitivity to $M_{A}$ allows a stringent test of the model.

Several analyses of the sensitivity to $M_{A}$ at the $\mathrm{LC}$ or the $\gamma \mathrm{C}$ have been carried out in the literature [43, 44, 45, 46, 100 (for an analysis focusing on the measurements with a GigaZ option of the LC see Ref. [101]). While in many of these analyses particular "benchmark" values of the SUSY parameters have been chosen, we perform a detailed scan over the parameter space of the three soft SUSY-breaking scenarios. This is in contrast to previous studies on the Higgs branching ratios in the literature [44, 45, 46], where all parameters except for the one under investigation have been kept fixed. In this case the assumed deviation between the MSSM and the SM is solely attributed to this single free parameter. This corresponds to a situation with a complete knowledge of all other SUSY parameters without any experimental or theoretical uncertainty, which obviously leads to an unrealistic enhancement of the sensitivity to the investigated parameter. Allowing the other SUSY (and SM) parameters to vary within reasonable ranges would result in reduced sensitivities as compared to the ones reported in these studies.

Since assumptions about which part of the SUSY spectrum might be accessible at the next generation of colliders are necessarily very speculative, we do not assume any further information beyond the Higgs sector at all and perform a full scan over the parameter space of the three soft SUSY-breaking scenarios. The resulting sensitivity to $M_{A}$ (which effectively covers also possible theoretical uncertainties ${ }^{6}$ ) can thus be interpreted as a "worst case" scenario within mSUGRA, mGMSB and mAMSB, which could be improved by incorporating further information from other sectors of the model.

In Fig. 4 the indirect sensitivity to $M_{A}$ within the mSUGRA scenario is investigated for the channels $h \rightarrow b \bar{b}, c \bar{c}, \tau^{+} \tau^{-}, W W^{*}$ at the LC. The figure shows the regions in the $M_{A}-\tan \beta$ plane where the result in the mSUGRA scenario differs from the SM prediction by $1 \sigma, 2 \sigma$ or $3 \sigma$, according to the prospective accuracy at the LC as given in Tab. 3. The corresponding sensitivities at the $\gamma \mathrm{C}$ (which are not shown here) turn out to be usually worse than at the LC for the mSUGRA scenario.

If a $2 \sigma$ or $3 \sigma$ deviation of the Higgs branching ratios from the corresponding SM values is found at the LC, an upper bound on $M_{A}$ can be inferred within the mSUGRA scenario

\footnotetext{
${ }^{6}$ Note that the presently largest theoretical uncertainty in the MSSM Higgs sector, which arises from the experimental error of the top-quark mass, will be drastically reduced by the precise measurement of $m_{t}$ at the LC.
} 

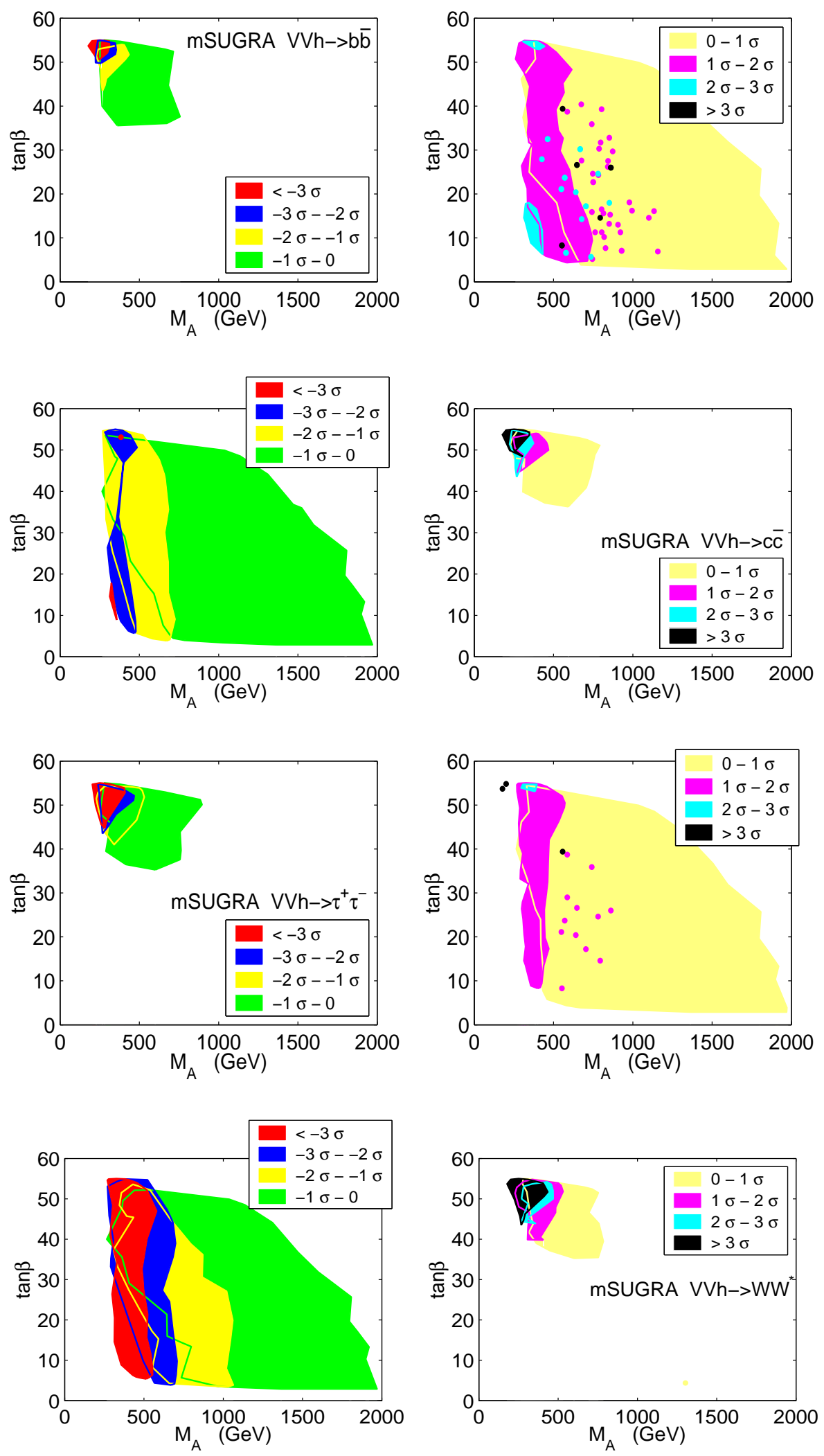

Figure 4: Indirect sensitivity to $M_{A}$ in the mSUGRA scenario: for the channels $h \rightarrow b \bar{b}$, $h \rightarrow c \bar{c}, h \rightarrow \tau^{+} \tau^{-}$and $h \rightarrow W W^{*}$ (from top to bottom) the regions in the $M_{A^{-}} \tan \beta$ plane are shown where the result in the mSUGRA scenario differs from the SM prediction by $1 \sigma$, $2 \sigma$ or $3 \sigma$, assuming the prospective accuracy at the LC according to Tab. 3 . 
according to Fig. [4. In particular, the $h \rightarrow W W^{*}$ channel yields an upper bound on $M_{A}$ of $500-600 \mathrm{GeV}$ (depending on $\tan \beta$ ) for a more than $3 \sigma$ deviation, $600-700 \mathrm{GeV}$ for a deviation in excess of $2 \sigma$, while deviations of more than $1 \sigma$ occur for $M_{A}$ up to $800-1000 \mathrm{GeV}$ within the mSUGRA scenario. On the other hand, measuring a suppression in the $h \rightarrow$ $b \bar{b}$ and/or $h \rightarrow \tau^{+} \tau^{-}$channel (left column of Fig. (4) or an enhancement in the $h \rightarrow c \bar{c}$ and/or $h \rightarrow W W^{*}$ channel (right column of Fig. (4) would determine $\tan \beta$ to lie within $35 \lesssim \tan \beta \lesssim 55$ in the mSUGRA scenario. The mSUGRA scenario is the only of the three soft SUSY-breaking scenarios that could accommodate a suppression of the $h \rightarrow b \bar{b}$ and/or $h \rightarrow \tau^{+} \tau^{-}$channel $^{7}$. Thus these measurements can help to distinguish the soft SUSY-breaking scenarios, see Sect. 4.3
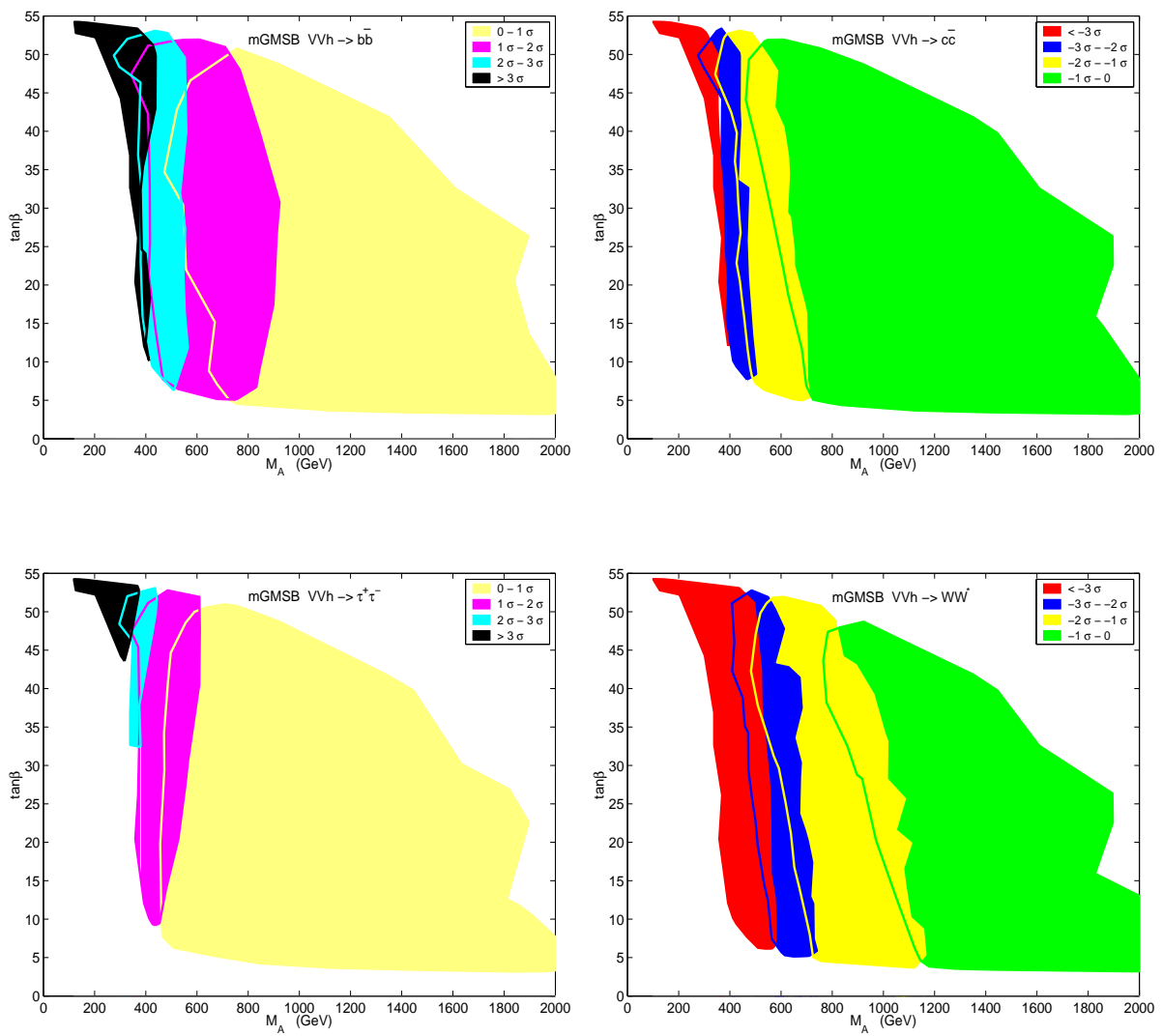

Figure 5: Indirect sensitivity to $M_{A}$ in the mGMSB scenario: for the channels $h \rightarrow b \bar{b}$ (top left), $h \rightarrow c \bar{c}$ (top right), $h \rightarrow \tau^{+} \tau^{-}$(bottom left) and $h \rightarrow W W^{*}$ (bottom right) the regions in the $M_{A}-\tan \beta$ plane are shown where the result in the mGMSB scenario differs from the SM prediction by $1 \sigma, 2 \sigma$ or $3 \sigma$, assuming the prospective accuracy at the $\mathrm{LC}$ according to Tab. [3.

In Fig. [5 we show the sensitivity to $M_{A}$ within the mGMSB scenario for the channels $h \rightarrow b \bar{b}, c \bar{c}, \tau^{+} \tau^{-}, W W^{*}$ at the LC. The corresponding results for $h \rightarrow b \bar{b}, W W^{*}$ at the $\gamma \mathrm{C}$ are displayed in Fig. [6 (which yields comparable sensitivities in this scenario). As for

\footnotetext{
${ }^{7}$ In our $\mathcal{O}(50000)$ mGMSB scatter points we have found two points with $M_{A} \approx 100 \mathrm{GeV}$ and $\tan \beta \approx 55$ that exhibit a very strong suppression of the $h \rightarrow b \bar{b}$ and $h \rightarrow \tau^{+} \tau^{-}$channel by more than $50 \%$. However, these points appear to be rather fine-tuned and we did not include them into our analysis.
} 

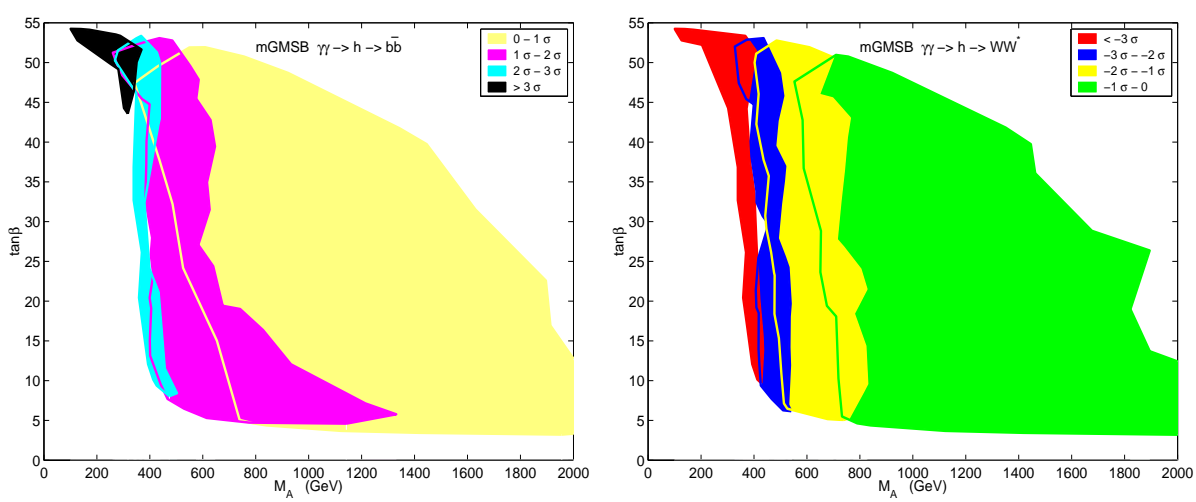

Figure 6: Indirect sensitivity to $M_{A}$ in the mGMSB scenario: for the channels $h \rightarrow b \bar{b}$ (left) and $h \rightarrow W W^{*}$ (right) the regions in the $M_{A}-\tan \beta$ plane are shown where the result in the mGMSB scenario differs from the SM prediction by $1 \sigma, 2 \sigma$ or $3 \sigma$, assuming the prospective accuracy at the $\gamma \mathrm{C}$ according to Tab. 3.
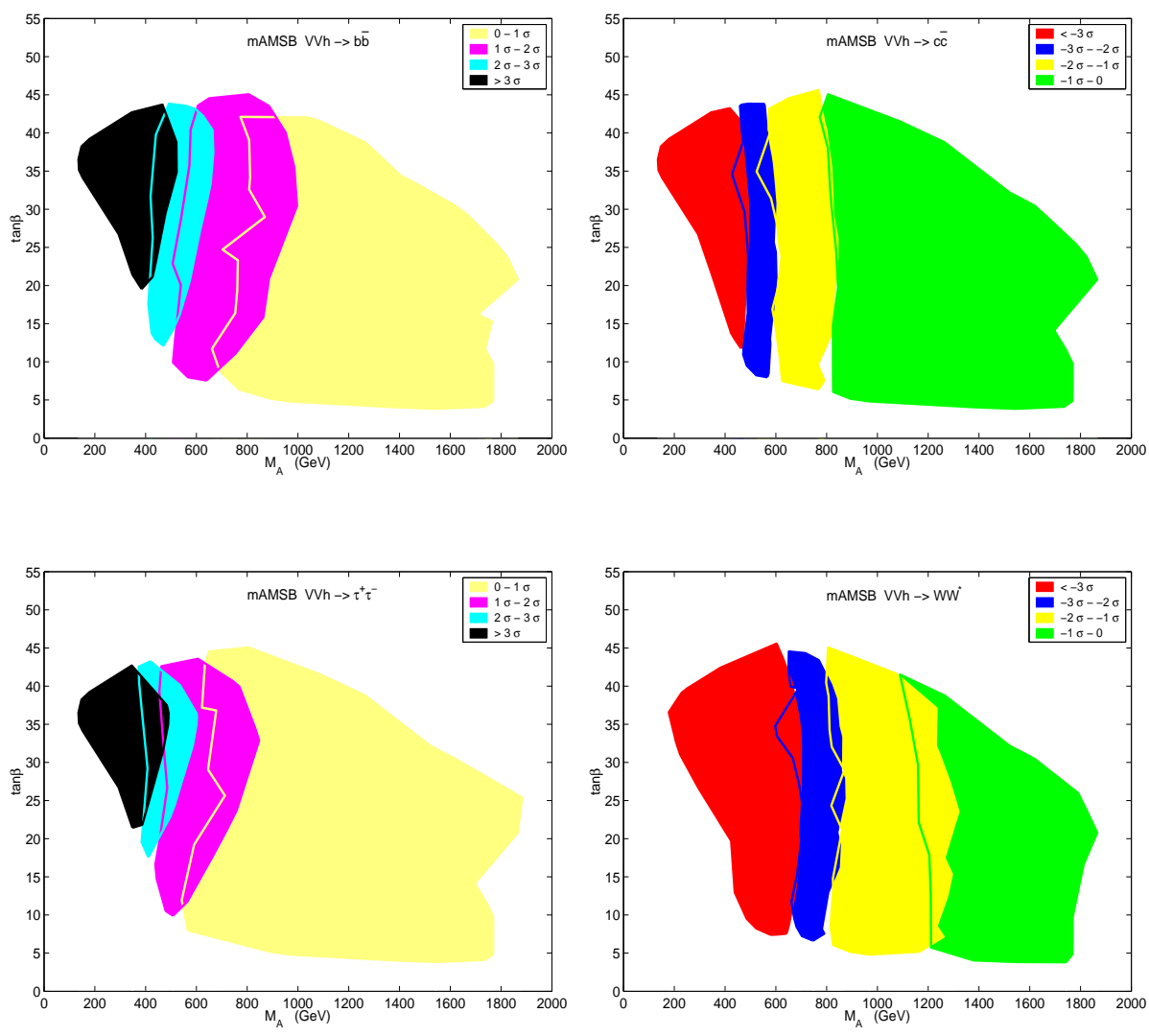

Figure 7: Indirect sensitivity to $M_{A}$ in the mAMSB scenario: for the channels $h \rightarrow b \bar{b}$ (top left), $h \rightarrow c \bar{c}$ (top right), $h \rightarrow \tau^{+} \tau^{-}$(bottom left) and $h \rightarrow W W^{*}$ (bottom right) the regions in the $M_{A}-\tan \beta$ plane are shown where the result in the mAMSB scenario differs from the SM prediction by $1 \sigma, 2 \sigma$ or $3 \sigma$, assuming the prospective accuracy at the LC according to Tab. 3 
mSUGRA, the observation of a $2 \sigma$ or $3 \sigma$ deviation compared to the SM prediction will allow to establish an upper bound on $M_{A}$ within the mGMSB scenario. Also in this case the $h \rightarrow W W^{*}$ channel shows significant deviations from the SM prediction over a wider range of the $M_{A}-\tan \beta$ plane than the other channels. For a more than $2 \sigma$ deviation in this channel an upper bound on $M_{A}$ of about $700 \mathrm{GeV}$ (depending somewhat on $\tan \beta$ ) can be inferred. Bigger deviations result in correspondingly lower upper bounds on $M_{A}$.

In Fig. [7 the sensitivity to $M_{A}$ within the mAMSB scenario is displayed for the channels $h \rightarrow b \bar{b}, c \bar{c}, \tau^{+} \tau^{-}, W W^{*}$ at the LC. The sensitivities at the $\gamma \mathrm{C}$ are usually worse in this scenario. As for the other two scenarios, in general an upper bound on $M_{A}$ can be established if a $2 \sigma$ or $3 \sigma$ deviation from the SM result is observed. Again in particular the $h \rightarrow$ $W W^{*}$ channel offers good prospects for observing sizable deviations. It allows to set an upper bound on $M_{A}$ of $800-900 \mathrm{GeV}$ (depending on $\tan \beta$ ) if a deviation of more than $2 \sigma$ is observed. Higher deviations result in correspondingly lower upper bounds on $M_{A}$. Comparing the results for the $h \rightarrow W W^{*}$ channel in the mAMSB scenario with the other scenarios, in the mAMSB scenario sizable deviations from the SM prediction occur over a wider parameter space in the $M_{A}-\tan \beta$ plane than in the other scenarios. Thus, the prospects for experimentally establishing a deviation from the SM prediction and in this way inferring an upper bound on $M_{A}$ appear to be particularly good in the mAMSB scenario.

\subsection{Sensitivity to high-energy parameters}

Besides providing sensitivity to $M_{A}$, precise measurements of Higgs branching rations at the LC can also yield indirect information on the high-energy parameters of the different soft SUSY-breaking scenarios. In Fig. 8 the results for the channels $h \rightarrow b \bar{b}, c \bar{c}, \tau^{+} \tau^{-}, W W^{*}$ are shown in the $m_{1 / 2}-m_{0}$ plane for the mSUGRA scenario. While the indirect constraints that can be obtained with a $2 \sigma$ or $3 \sigma$ deviation on $m_{0}$ are rather mild, stronger bounds can be obtained for $m_{1 / 2}$. This reflects the fact that $M_{A}$ and the squark masses are strongly correlated with the $m_{1 / 2}$ value. Combining the channels, an upper bound of $\sim 350 \mathrm{GeV}$ on $m_{1 / 2}$ can be set if a deviation of more than $3 \sigma$ from the SM prediction is observed, a $2 \sigma$ deviation constrains $m_{1 / 2}$ to be smaller than $\sim 450 \mathrm{GeV}$, while deviations of more than $1 \sigma$ occur for $m_{1 / 2} \lesssim 650 \mathrm{GeV}$.

Concerning the mGMSB scenario (which is not displayed here) the indirect constraints are weaker. Results deviating from the SM prediction for the $h \rightarrow W W^{*}$ channel by $3 \sigma$, for instance, are distributed over nearly the whole $M_{\text {mess }}-\Lambda$ plane. Thus, establishing a non SM-like behavior in the Higgs sector alone is not sufficient to derive indirect bounds on $M_{\text {mess }}$ and $\Lambda$, further experimental information is necessary to constrain these parameters. On the other hand, weak lower limits on $M_{\text {mess }}, \Lambda$ could be set, which can cut out the lower edge of the mGMSB allowed $M_{\text {mess }}-\Lambda$ area, if the deviation from the SM value is found to be small.

In Fig. 9 the results for the channels $h \rightarrow b \bar{b}, c \bar{c}$ are shown in the $m_{0}-m_{\text {aux }}$ plane for the mAMSB scenario. Like in the mGMSB scenario, deviations of $3 \sigma$ or $2 \sigma$ with respect to the SM prediction occur over a rather wide range of $m_{0}$ and $m_{\text {aux }}$ values. An observed deviation of $3 \sigma$ would constrain $m_{0}$ to be smaller than $\sim 1100 \mathrm{GeV}$, while $m_{\text {aux }}$ would have to be smaller than $\sim 6 \cdot 10^{4} \mathrm{GeV}$. Observation of a $2 \sigma$ deviation would allow to set an

upper bound on $m_{0}$ of $m_{0} \lesssim 1400 \mathrm{GeV}$, while restricting the parameter space to the one compatible with a $2 \sigma$ deviation does not significantly reduce the range of possible values of $m_{\text {aux }}$ in Fig. 9] 

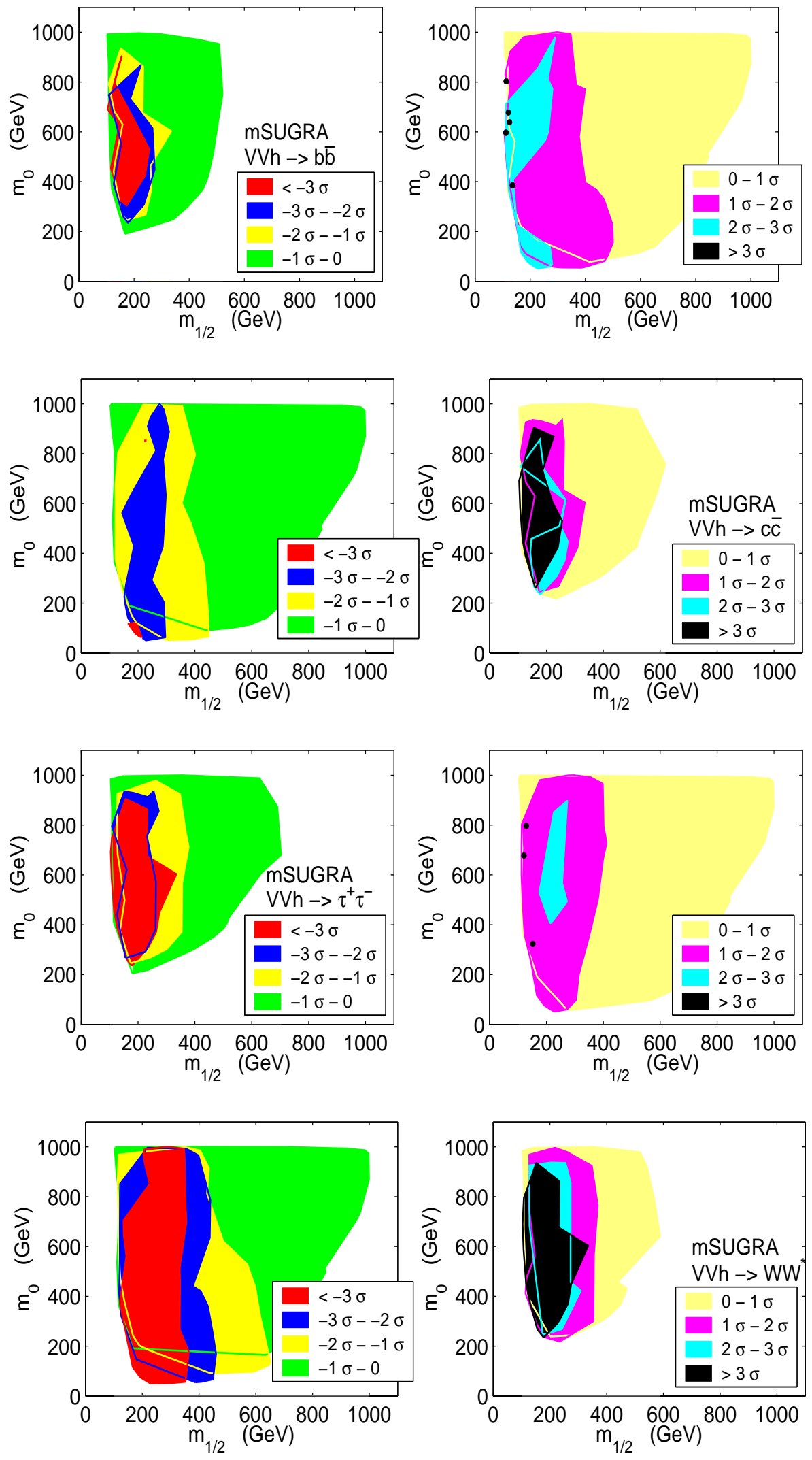

Figure 8: Indirect sensitivity to $m_{0}, m_{1 / 2}$ in the mSUGRA scenario: for the channels $h \rightarrow b \bar{b}$, $h \rightarrow c \bar{c}, h \rightarrow \tau^{+} \tau^{-}$and $h \rightarrow W W^{*}$ (from top to bottom) the regions in the $m_{1 / 2}-m_{0}$ plane are shown where the result in the mSUGRA scenario differs from the SM prediction by $1 \sigma$, $2 \sigma$ or $3 \sigma$, assuming the prospective accuracy at the LC according to Tab. 3 . 

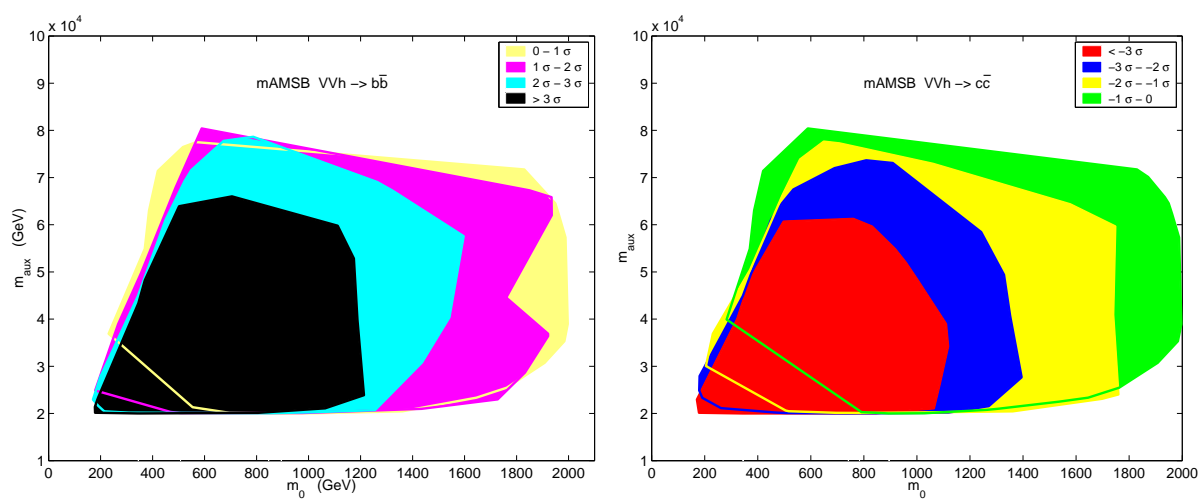

Figure 9: Indirect sensitivity to $m_{0}, m_{\text {aux }}$ in the mAMSB scenario: for the channels $h \rightarrow b \bar{b}$ (left) and $h \rightarrow c \bar{c}$ (right) the regions in the $m_{0}-m_{\text {aux }}$ plane are shown where the result in the mAMSB scenario differs from the SM prediction by $1 \sigma, 2 \sigma$ or $3 \sigma$, assuming the prospective accuracy at the LC according to Tab. 3 .

\subsection{Discrimination between soft SUSY-breaking scenarios}

We now investigate the potential of precise measurements of Higgs branching ratios at a LC for distinguishing between the three soft SUSY-breaking scenarios. The main experimental test of different SUSY-breaking scenarios will of course be to confront their predictions for the SUSY spectrum with the results obtained from the direct observation of SUSY particles. The analysis of the Higgs sector, however, may contribute further valuable information. Since the different soft SUSY-breaking scenarios predict different mass patterns for the SUSY particles, variations in the results for the Higgs sector observables for the same value of $M_{A}$ can be expected.

As starting point of our analysis, as above, we do not assume experimental input from other sectors of the MSSM, but concentrate on the Higgs sector. Clearly, resolving differences between the predictions of the three scenarios via measurements in the Higgs sector will require some experimental information on the tree-level parameters of the Higgs sector, $M_{A}$ and $\tan \beta$. Therefore we will focus on a scenario where the LHC can detect the heavy MSSM Higgs bosons via their decays $H / A \rightarrow \tau^{+} \tau^{-}$(with the main production channel $b \bar{b} \rightarrow b \bar{b} H / A$ ), which can be realized for large $\tan \beta$ and not too large $M_{A}$ [77, 102]. As a specific example we assume that the LHC provides a measurement of $M_{A}$ as well as a lower bound on $\tan \beta$,

$$
500 \mathrm{GeV} \lesssim M_{A} \lesssim 600 \mathrm{GeV}, \quad \tan \beta \gtrsim 30
$$

The results of the analysis below would improve for smaller values of $M_{A}$, while for a larger $M_{A}$ and smaller $\tan \beta$ observation of the heavy Higgs bosons at the LHC would become increasingly difficult. Restricting the data set of our scan, see Sect. 2.1, to those parameter points fulfilling Eq. (25) we compare the predictions for the different branching ratios arising from the three scenarios. As above, we indicate the deviations from the SM prediction in terms of the prospective accuracy at the LC according to Tab. 3 .

In Fig. 10 we show the results for the channels $h \rightarrow b \bar{b}$ and $h \rightarrow g g$. The results for these channels, as for the others that are not shown $\left(h \rightarrow \tau^{+} \tau^{-}, h \rightarrow c \bar{c}\right.$ and $\left.h \rightarrow W W^{*}\right)$, are similar and show the following general pattern (see also the discussion in Sect. 3 and Sect. 4.1): for the $M_{A}$ values corresponding to Eq. (25) the mAMSB scenario gives rise 

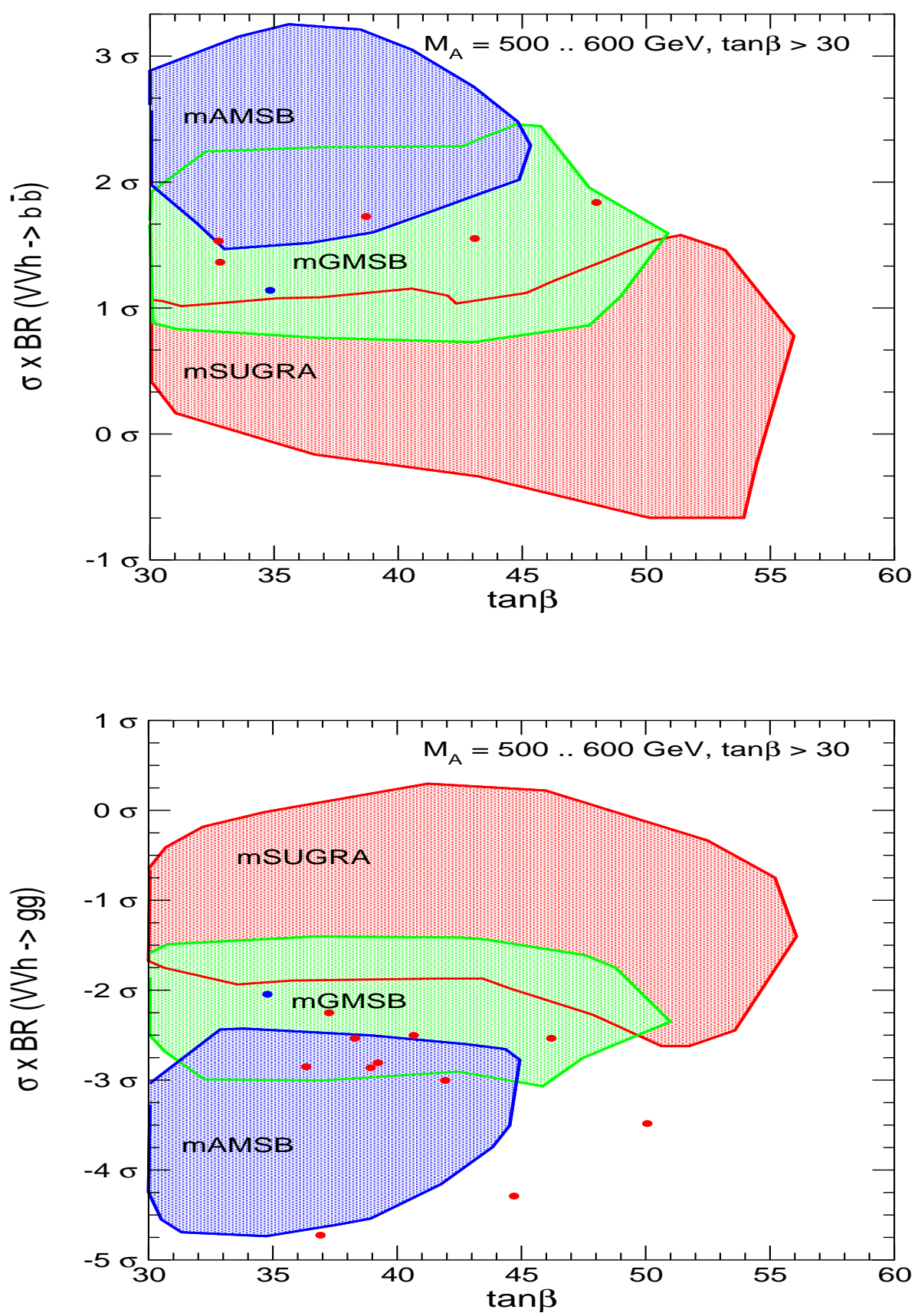

Figure 10: Comparison of $\mathrm{BR}(h \rightarrow b \bar{b})$ (top) and $\mathrm{BR}(h \rightarrow g g)$ (bottom) in the three soft SUSY-breaking scenarios via LC measurements. 
to larger deviations in the branching ratios from the SM values than the mGMSB scenario. Thus, if in the situation of Eq. (25) a $3 \sigma$ deviation from the SM value were found in $\mathrm{BR}(h \rightarrow$ $b \bar{b})$ and $\mathrm{a}-4 \sigma$ deviation in $\operatorname{BR}(h \rightarrow g g)$, this would be better compatible with an AMSB scenario than with a mGMSB scenario. If, on the other hand, the branching ratios were found to agree well with the SM prediction, this would be best compatible with a SUSY-breaking scenario of mSUGRA type.

As a consequence, precision measurements at the LC of the branching ratios of the light $\mathcal{C P}$-even Higgs boson of the MSSM may indicate a preference among the three soft SUSY-breaking scenarios at the 1-2 $\sigma$ level. This information will be complementary to the information from the direct observation of SUSY particles.

The different behavior as a function of $M_{A}$ in the three scenarios can be traced back mainly to different loop contributions to the off-diagonal entry in the Higgs propagator matrix, $\hat{\Sigma}_{\phi_{1} \phi_{2}}$, which according to Eq. (7) give rise to differences in the effective mixing angle $\alpha_{\text {eff }}$ entering the Higgs couplings. Especially the dominant decay channel $h \rightarrow b \bar{b}$, being $\sim \sin ^{2} \alpha_{\text {eff }} / \cos ^{2} \beta$, is strongly affected. While in the mSUGRA scenario $\hat{\Sigma}_{\phi_{1} \phi_{2}}$ has in general fairly large and negative values, in the mGMSB scenario $\hat{\Sigma}_{\phi_{1} \phi_{2}}$ is small, and in the mAMSB scenario it gets large and positive values. In combination with the tree-level dependence on $M_{A}$, see Eq. (7), this leads to a different degree of decoupling with respect to the SM result as function of $M_{A}$.

So far we have not assumed any additional experimental input on the SUSY spectrum from the Tevatron or the LHC. We have checked, however, that the results in Fig. 10] are essentially unmodified if parameter points for which the Tevatron will detect SUSY particles are excluded from the scan.

Concerning possible experimental information on the SUSY spectrum from the LHC, the situation strongly depends on the assumed scenario. For illustration we thus restrict to one particular example, shown in Fig. 11] The regions indicated by dashed lines correspond to parameter regions in the three scenarios where experimental information on the light scalar top quark is assumed,

$$
800 \mathrm{GeV} \lesssim m_{\tilde{t}_{1}} \lesssim 900 \mathrm{GeV} .
$$

The shaded areas surrounded by full lines correspond to the case where furthermore the gluino mass is assumed to be bounded by

$$
900 \mathrm{GeV} \lesssim m_{\tilde{g}} \lesssim 1000 \mathrm{GeV}
$$

As expected, assuming direct experimental information on the SUSY spectrum in addition to measurements in the Higgs sector significantly enhances the sensitivity for distinguishing between the different soft SUSY-breaking scenarios. While for the particular scenario studied here it is not possible to distinguish between the mGMSB and mSUGRA scenarios on the basis of the Higgs branching ratios alone, additional information on $\tan \beta$ would allow a clear distinction.

\section{Conclusions}

We have investigated the relevant production and decay channels of the lightest $\mathcal{C P}$-even MSSM Higgs boson at the Tevatron, the LHC, an $e^{+} e^{-} \mathrm{LC}$, a $\gamma \mathrm{C}$ and a $\mu \mathrm{C}$ within the 

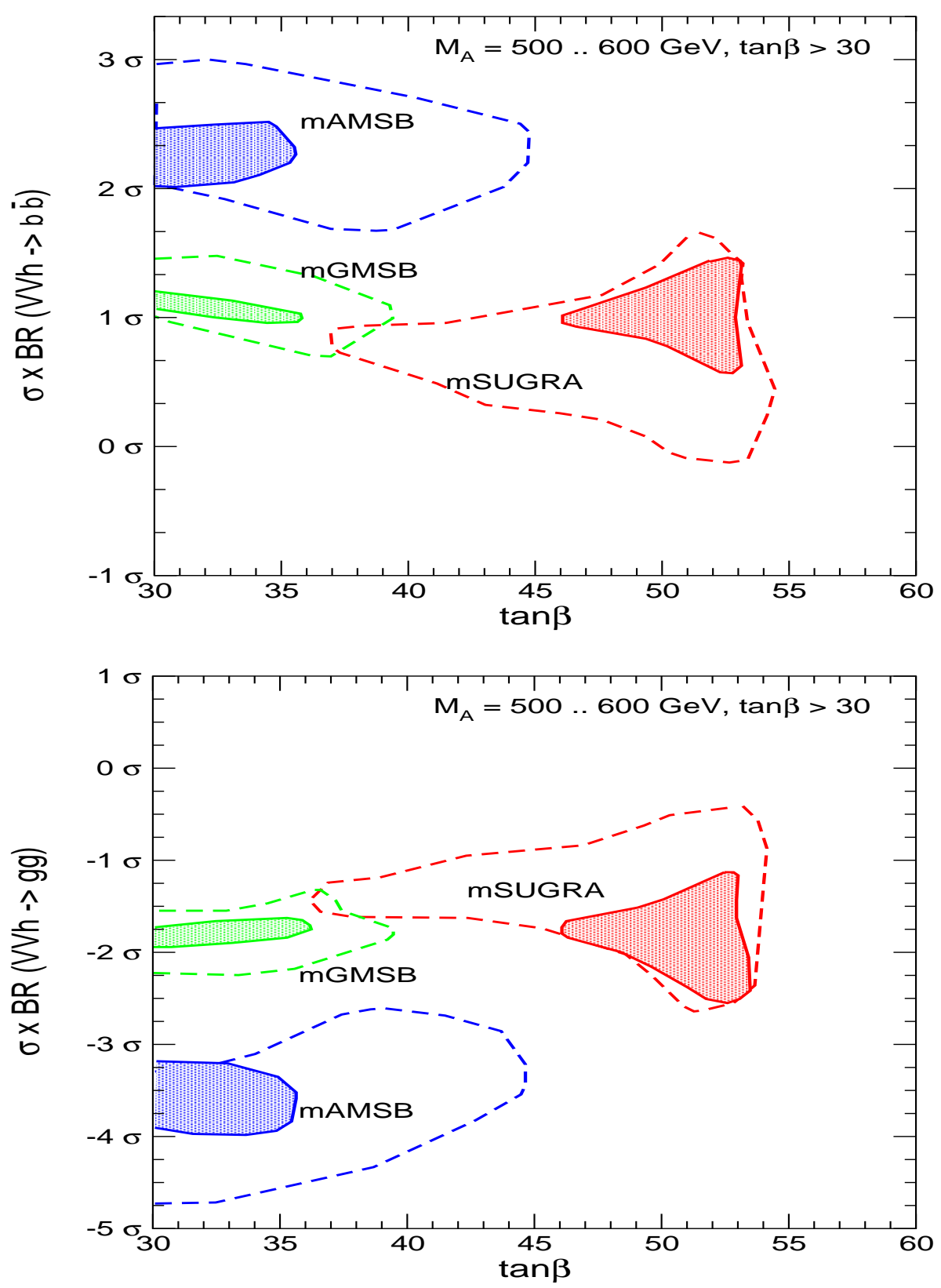

Figure 11: Comparison of $\operatorname{BR}(h \rightarrow b \bar{b})$ (top) and $\operatorname{BR}(h \rightarrow g g)$ (bottom) in the three soft SUSY-breaking scenarios via LC measurements, assuming direct input on the SUSY spectrum from the LHC. The areas surrounded by dashed lines correspond to the parameter regions in the three scenarios where the light scalar top mass lies in the region $800 \mathrm{GeV} \leq$ $m_{\tilde{t}_{1}} \leq 900 \mathrm{GeV}$, while the shaded areas surrounded by full lines correspond to the case where furthermore the gluino mass is known to be constrained by $900 \mathrm{GeV} \leq m_{\tilde{g}} \leq 1000 \mathrm{GeV}$. 
mSUGRA, mGMSB and mAMSB scenarios. The values of $\sigma \times$ BR have been compared with the corresponding SM values with the same Higgs boson mass, $M_{H_{\mathrm{SM}}}=m_{h}$. In this context we have also updated earlier results on the upper bound on $m_{h}$ within the three scenarios and on the lower bounds on $\tan \beta$ that can be inferred by confronting the theoretical predictions with the LEP exclusion limit.

We have first analyzed the observability of the lightest MSSM Higgs boson at the different colliders. The modes $g g \rightarrow h \rightarrow \gamma \gamma, t \bar{t} \rightarrow t \bar{t} h$ and $W W \rightarrow h \rightarrow W W^{*}, \tau^{+} \tau^{-}, \gamma \gamma$ allow the detection of the lightest MSSM Higgs boson in all three scenarios over the whole corresponding parameter space. Possible exceptions occur for the very small $M_{A}$ region in the mGMSB and mAMSB scenarios, where a strong suppression of more than $50 \%$ could happen for $g g \rightarrow h \rightarrow \gamma \gamma$. Within the clean experimental environment of the LC the observation of the light Higgs will be ensured for all three scenarios. For a $\gamma \mathrm{C}$, the very small $M_{A}$ region in mGMSB and mAMSB can be problematic for the $h \rightarrow b \bar{b}$ and $h \rightarrow \tau^{+} \tau^{-}$mode. At the $\mu \mathrm{C}$, $M_{A} \lesssim 150 \mathrm{GeV}$ and $\tan \beta \gtrsim 50$ for mSUGRA exhibits a strong suppression for the $h \rightarrow b \bar{b}$ and $h \rightarrow \tau^{+} \tau^{-}$mode, while on the other hand in this parameter region the production of the heavy MSSM Higgs bosons $H, A$ happens with an enhanced rate. Besides these difficult regions, the main search modes at a $\gamma \mathrm{C}$ and a $\mu \mathrm{C}$ do not suffer from severe suppressions with respect to the SM case in all three scenarios. The results of this analysis are summarized in Tab. 2. Thus, all possible future colliders offer very good prospects for detecting the lightest $\mathcal{C P}$-even Higgs boson of the MSSM in an mSUGRA, mGMSB or mAMSB scenario.

We then investigated the potential of precision measurements of Higgs branching ratios at the $\mathrm{LC}$ and the $\gamma \mathrm{C}$ for establishing indirect constraints on $M_{A}$ and $\tan \beta$. For this analysis we have not assumed any further experimental information on the SUSY spectrum, i.e. a full scan over the parameter space (restricting to the case $\mu>0$ ) has been performed. If

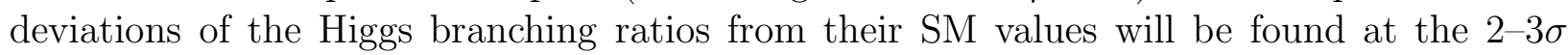
level, it will be possible to establish an upper bound for $M_{A}$ significantly below $1 \mathrm{TeV}$ in all three scenarios. The biggest sensitivity will come from the $h \rightarrow W W^{*}$ and $h \rightarrow c \bar{c}$ channels. Within the mSUGRA scenario, furthermore a bound on $\tan \beta$ of $35 \lesssim \tan \beta \lesssim 55$ can be obtained if a suppression of the $h \rightarrow b \bar{b}$ and/or $h \rightarrow \tau^{+} \tau^{-}$channel or an enhancement in the $h \rightarrow c \bar{c}$ and/or $h \rightarrow W W^{*}$ channel with respect to the SM values is observed. If this would be the case, this could be independently confirmed by Higgs mediated B-physics observables like $B^{0} \rightarrow \mu^{+} \mu^{-}$or $B^{0}-\bar{B}^{0}$ mixing.

Similarly, precise measurements of $\sigma \times \mathrm{BR}$ at the $\mathrm{LC}$ can also provide indirect information on the high-energy parameters of the three soft SUSY-breaking scenarios. While within the mGMSB scenario the experimental determination of the Higgs branching ratios will allow to set only very weak bounds on the high-energy parameters, within mSUGRA relatively strong bounds on $m_{1 / 2}$ and in mAMSB moderate bounds on $m_{0}$ could be set.

Finally we have investigated the potential of precise measurements of $\sigma \times \mathrm{BR}$ at a LC to distinguish between the three soft SUSY-breaking scenarios. For this analysis we have assumed a situation where experimental information on $M_{A}$ (and to a lesser extent on $\tan \beta$ ) obtained at the LHC can be combined with precision measurements of the properties of the light Higgs boson at the LC, see also Ref. 103. If a significant suppression of the $h \rightarrow b \bar{b}$ and/or $h \rightarrow \tau^{+} \tau^{-}$channel with respect to its SM value were found, this would point towards the mSUGRA scenario, irrespectively of the actual value of $M_{A}$ (with $M_{A} \lesssim 1 \mathrm{TeV}$ ). Otherwise, assuming in our example $M_{A}$ to be restricted to $500 \mathrm{GeV} \lesssim M_{A} \lesssim 600 \mathrm{GeV}$, precise measurements of $\sigma \times \mathrm{BR}$ in particular in the $h \rightarrow b \bar{b}$ and $h \rightarrow g g$ channels may 
indicate a preference among the three soft SUSY-breaking scenarios at the $1-2 \sigma$ level. This information might be valuable as it complements the one about the SUSY spectrum from the direct observation of SUSY particles.

\section{Acknowledgements}

We thank S. Ambrosanio for helpful discussion on the GMSB scenario as well as for his Fortran code SUSYFIRE. We thank T. Plehn for discussions. A.D. thanks P. Slavich for interesting discussions. S.S. has been supported by the DOE grant DE-FG03-92-ER-40701. A.D. would like to acknowledge financial support from the Network RTN European Program HPRN-CT-2000-00148 "Physics Across the Present Energy Frontier: Probing the Origin of Mass". This work has been supported by the European Community's Human Potential Programme under contract HPRN-CT-2000-00149 Physics at Colliders.

\section{References}

[1] G. Kane, C. Kolda and J. Wells, Phys. Rev. Lett. 70 (1993) 2686, hep-ph/9210242

J. Espinosa and M. Quirós, Phys. Lett. B 302 (1993) 51, hep-ph/9212305

[2] [LEP Higgs working group], Phys. Lett. B 565 (2003) 61, hep-ex/0306033.

[3] S. Heinemeyer, W. Hollik and G. Weiglein, Eur. Phys. Jour. C 9 (1999) 343, hep-ph/9812472.

[4] G. Degrassi, S. Heinemeyer, W. Hollik, P. Slavich and G. Weiglein, to appear in Eur. Phys. Jour. C, hep-ph/0212020.

[5] S. Heinemeyer, W. Hollik and G. Weiglein, Phys. Rev. D 58 (1998) 091701, hep-ph/9803277, Phys. Lett. B 440 (1998) 296, hep-ph/9807423.

[6] M. Carena, J. Espinosa, M. Quirós and C. Wagner, Phys. Lett. B 355 (1995) 209, hep-ph/9504316.

[7] M. Carena, M. Quirós and C. Wagner, Nucl. Phys. B 461 (1996) 407, hep-ph/9508343.

[8] H. Haber, R. Hempfling and A. Hoang, Z. Phys. C 75 (1997) 539, hep-ph/9609331.

[9] R. Hempfling and A. Hoang, Phys. Lett. B 331 (1994) 99, hep-ph/9401219

[10] R. Zhang, Phys. Lett. B 447 (1999) 89, hep-ph/9808299.

[11] J. Espinosa and R. Zhang, Nucl. Phys. B 586 (2000) 3, hep-ph/0003246

[12] J. Espinosa and I. Navarro, Nucl. Phys. B 615 (2001) 82, hep-ph/0104047.

[13] G. Degrassi, P. Slavich and F. Zwirner, Nucl. Phys. B 611 (2001) 403, hep-ph/0105096; A. Brignole, G. Degrassi, P. Slavich and F. Zwirner, Nucl. Phys. B 631 (2002) 195, hep-ph/0112177; Nucl. Phys. B 643 (2002) 79, hep-ph/0206101. 
[14] S. Martin, Phys. Rev. D 65 (2002) 116003, hep-ph/0111209 Phys. Rev. D 66 (2002) 096001, hep-ph/0206136 hep-ph/0211366.

[15] M. Carena, S. Heinemeyer, C. Wagner and G. Weiglein, hep-ph/9912223

[16] M. Carena, S. Heinemeyer, C. Wagner and G. Weiglein, Eur. Phys. Jour. C 26 (2003) 601, hep-ph/0202167.

[17] B. Allanach et al., Eur. Phys. J. C 25 (2002) 113, hep-ph/0202233.

[18] H. Nilles, Phys. Lett. B 115 (1982) 193; Nucl. Phys. B 217 (1983) 366.

A. Chamseddine, R. Arnowitt and P. Nath, Phys. Rev. Lett. 49 (1982) 970;

R. Barbieri, S. Ferrara and C. Savoy, Phys. Lett. B 119 (1982) 343;

H. Nilles, M. Srednicki and D. Wyler, Phys. Lett. B 120 (1983) 346.

E. Cremmer, P. Fayet and L. Girardello, Phys. Lett. B 122 (1983) 41.

S. Ferrara, L. Girardello and H. Nilles, Phys. Lett. B 125 (1983) 457. L. Hall, J. Lykken and S. Weinberg, Phys. Rev. D 27 (1983) 2359;

S. Soni and H. Weldon, Phys. Lett. B 126 (1983) 215.

R. Arnowitt, A. Chamseddine and P. Nath, Nucl. Phys. B 227 (1983) 121.

For more details see, S. Weinberg, "The quantum theory of fields. Vol. 3: Supersymmetry," Cambridge University Press (2000).

[19] For reviews see also:

H. Nilles, Phys. Rept. 110 (1984) 1;

H. Haber and G. Kane, Phys. Rept. 117 (1985) 75;

A. Lahanas and D. Nanopoulos, Phys. Rept. 145 (1987) 1;

S. Martin, in "Perspectives on supersymmetry", ed. G. Kane, hep-ph/9709356, see: zippy.physics.niu.edu/primer.shtml.

[20] For a review, see: G.F. Giudice and R. Rattazzi, Phys. Rept. 322 (1999) 419, hep-ph/9801271.

[21] L. Randall and R. Sundrum, Nucl. Phys. B 557 (1999) 79, hep-th/9810155.

[22] G.F. Giudice, M.A. Luty, H. Murayama and R. Rattazzi, JHEP 9812 (1998) 027, hep-ph/9810442.

[23] T. Gherghetta, G.F. Giudice, J.D. Wells, Nucl. Phys. B 559 (1999) 27, hep-ph/9904378.

[24] K. Matchev and D. Pierce, Phys. Lett. B 445 (1999) 331, hep-ph/9805275;

W. de Boer, hep-ph/9808448.

[25] T. Kaeding and S. Nandi, hep-ph/9906342.

[26] S. Su, Nucl. Phys. B 573 (2000) 87, hep-ph/9910481.

[27] B. Allanach and A. Dedes, JHEP 0006 (2000) 017, hep-ph/0003222.

[28] A. Dedes, S. Heinemeyer, P. Teixeira-Dias and G. Weiglein, Jour. Phys. G 26 (2000) 582, hep-ph/9912249. 
[29] S. Ambrosanio, S. Heinemeyer and G. Weiglein, in hep-ph/0002191 and hep-ph/0005142.

[30] J. Ellis, G. Ganis, D. Nanopoulos and K. Olive, Phys. Lett. B 502 (2001) 171, hep-ph/0009355.

[31] A. Djouadi, M. Drees and J. Kneur, JHEP 0108 (2001) 055, hep-ph/0107316

[32] S. Ambrosanio, A. Dedes, S. Heinemeyer, S. Su and G. Weiglein, Nucl. Phys. B 624 (2001) 3, hep-ph/0106255,

A. Dedes, S. Heinemeyer, S. Su and G. Weiglein, hep-ph/0110219

[33] S. Martin and M. Vaughn, Phys. Rev. D 50 (1994) 2282, hep-ph/9311340;

Y. Yamada, Phys. Rev. D 50 (1994) 3537, hep-ph/9401241;

I. Jack and D. Jones, Phys. Lett. B 333 (1994) 372, hep-ph/9405233.

[34] B. Allanach, S. Kraml and W. Porod, JHEP 0303 (2003) 016, hep-ph/0302102.

[35] M. Carena, H. Haber, S. Heinemeyer, W. Hollik, C. Wagner and G. Weiglein, Nucl. Phys. B 580 (2000) 29, hep-ph/0001002.

[36] S. Heinemeyer, W. Hollik and G. Weiglein, hep-ph/9910283.

[37] J. Espinosa and R. Zhang, JHEP 0003 (2000) 026, hep-ph/9912236.

[38] S. Heinemeyer, W. Hollik and G. Weiglein, Comp. Phys. Comm. 1242000 76, hep-ph/9812320 hep-ph/0002213.

The codes are accessible via www. feynhiggs . de .

[39] M. Frank, S. Heinemeyer, W. Hollik and G. Weiglein, hep-ph/0202166.

[40] An advanced (and so far unpublished) version of FeynHiggs contains also the evaluation of the Higgs boson decay rates, combining the results from Refs. 41, 42.

[41] S. Heinemeyer, W. Hollik and G. Weiglein, Eur. Phys. Jour. C 16 (2000) 139, hep-ph/0003022.

[42] A. Djouadi, J. Kalinowski and M. Spira, Comput. Phys. Commun. 108 (1998) 56, hep-ph/9704448.

[43] J. Ellis, S. Heinemeyer, K. Olive and G. Weiglein, Phys. Lett. B 515 (2001) 348, hep-ph/0105061; JHEP 0301 (2003) 006, hep-ph/0211206.

[44] D. Asner et al., Eur. Phys. Jour. C 28 (2003) 27, hep-ex/0111056.

[45] J. Guasch, W. Hollik and S. Peñaranda, Phys. Lett. B 515 (2001) 367, hep-ph/0106027.

[46] M. Carena, H. Haber, H. Logan and S. Mrenna, Phys. Rev. D 65 (2002) 055005, E: ibid D 65 (2002) 099902, hep-ph/0106116. 
[47] H. Dreiner, hep-ph/9707435.

G. Bhattacharyya, hep-ph/9709395.

B. Allanach, A. Dedes and H. Dreiner, Phys. Rev. D 60 (1999) 075014, hep-ph/9906209.

[48] Part. Data Group, Phys. Rev. D 66 (2002) 010001.

[49] H. Brown et al. [Muon $g_{\mu}-2$ Collaboration], Phys. Rev. Lett. 86 (2001) 2227, hep-ex/0102017; updated as hep-ex/0208001;

A. Czarnecki and W. Marciano, Phys. Rev. D 64 (2001) 013014, hep-ph/0102122;

M. Davier, S. Eidelman, A. Hocker and Z. Zhang, hep-ph/0208177.

[50] S. Chen et al. [CLEO Collaboration], Phys. Rev. Lett. 87 (2001) 251807, hep-ex/0108032;

Y. Ushiroda [Belle Collaboration], hep-ex/0104045,

B. Aubert et al. [BaBar Collaboration], hep-ex/0207076.

[51] A. Dedes, A. Lahanas and K. Tamvakis, Phys. Rev. D 53, 3793 (1996), hep-ph/9504239.

[52] A. Dedes, S. Heinemeyer and G. Weiglein, "FeynSSG: A program for the minimal supergravity and Higgs spectrum", in

G. Azuelos et al., [Les Houches Beyond the SM working group], Summary report, hep-ph/0204031.

[53] M. Dine, A. Nelson, Phys. Rev. D 48 (1993) 1277, hep-ph/9303230;

M. Dine, A. Nelson, Y. Shirman, Phys. Rev. D 51 (1995) 1362, hep-ph/9408384.

M. Dine, A. Nelson, Y. Nir, Y. Shirman, Phys. Rev. D 53 (1996) 2658, hep-ph/9507378.

[54] M. Dine, W. Fischler, M. Srednicki, Nucl. Phys. B 189 (1981) 575;

S. Dimopoulos, S. Raby, Nucl. Phys. B 192 (1981) 353;

M. Dine, W. Fischler, Phys. Lett. B 110 (1982) 227;

M. Dine, M. Srednicki, Nucl. Phys. B 202 (1982) 238;

M. Dine, W. Fischler, Nucl. Phys. B 204 (1982) 346;

L. Alvarez-Gaumé, M. Claudson, M. Wise, Nucl. Phys. B 207 (1982) 96;

C. Nappi, B. Ovrut, Phys. Lett. B 113 (1982) 175;

S. Dimopoulos, S. Raby, Nucl. Phys. B 219 (1983) 479.

[55] P. Fayet, Phys. Lett. B 70 (1977) 461; Phys. Lett. B 86 (1979) 272; Phys. Lett. B 175 (1986) 471; and in "Unification of the fundamental particle interactions", eds. S. Ferrara, J. Ellis, P. van Nieuwenhuizen (Plenum, New York, 1980) p. 587.

[56] An updated, generalized and Fortran-linked version of the program used in Ref. [57. It generates minimal and non-minimal GMSB and SUGRA models. For inquiries about this software package, please send e-mail to Sandro.Ambrosanio@bancaroma.it.

[57] S. Ambrosanio, G. Kribs, S. Martin, Phys. Rev. D 56 (1997) 1761, hep-ph/9703211

[58] S. Ambrosanio, G. Blair, Eur. Phys. Jour. C 12 (2000) 287, hep-ph/9905403.

[59] S. Ambrosanio et al., JHEP 0101 (2001) 014, hep-ph/0010081. 
[60] A. Pomarol and R. Rattazzi, JHEP 9905 (1999) 013, hep-ph/9903448

[61] Z. Chacko, M. Luty and E. Ponton, JHEP 0004 (2000) 001, hep-ph/9905390.

[62] E. Katz, Y. Shadmi and Y. Shirman, JHEP 9908 (1999) 015, hep-ph/9906296.

[63] I. Jack, D. Jones and R. Wild, Phys. Lett. B 535, 193 (2002), hep-ph/0202101.

[64] L.J. Hall, R. Rattazzi and U. Sarid, Phys. Rev. D 50 (1994) 7048, hep-ph/9306309.

[65] M. Carena, S. Mrenna and C. Wagner, Phys. Rev. D 60 (1999) 075010, hep-ph/9808312; Phys. Rev. D 62 (2000) 055008, hep-ph/9907422.

H. Eberl, K. Hidaka, S. Kraml, W. Majerotto and Y. Yamada, Phys. Rev. D 62 (2000) 055006, hep-ph/9912463

M. Carena, D. Garcia, U. Nierste and C. Wagner, Nucl. Phys. B 577 (2000) 88, hep-ph/9912516.

[66] S. Heinemeyer, W. Hollik, J. Rosiek and G. Weiglein, Eur. Phys. Jour. C 19 (2001) 535, hep-ph/0102081.

[67] T. Hahn, S. Heinemeyer and G. Weiglein, Nucl. Phys. B 652 (2003) 229, hep-ph/0211204.

[68] H. Eberl, W. Majerotto, and V. Spanos, Phys. Lett. B 538 (2002) 353, hep-ph/0204280.

[69] S. Dawson, A. Djouadi and M. Spira, Phys. Rev. Lett. 77 (1996) 16, hep-ph/9603423.

[70] [LEP Higgs Working Group Collaboration], hep-ex/0107030; LHWG Note 2001-4, see: lephiggs . web.cern.ch/LEPHIGGS/papers/.

[71] R. Barate et al. [ALEPH Collaboration], Phys. Lett. B 499 (2001) 53, hep-ex/0010062.

[72] M. Veltman, Nucl. Phys. B 123 (1977) 89.

[73] A. Djouadi, P. Gambino, S. Heinemeyer, W. Hollik, C. Jünger and G. Weiglein, Phys. Rev. Lett. 78 (1997) 3626, hep-ph/9612363 Phys. Rev. D 57 (1998) 4179, hep-ph/9710438,

S. Heinemeyer and G. Weiglein, in Proc. of the 5th International Symposium on Radiative Corrections (RADCOR 2000) ed. H. Haber, hep-ph/0102317.

For a recent update, see also: S. Heinemeyer and G. Weiglein, JHEP 0210 (2002) 072, hep-ph/0209305: hep-ph/0301062.

[74] A. Dedes and P. Slavich, hep-ph/0212132.

[75] J. Ellis, J. Hagelin, D. Nanopoulos, K. Olive and M. Srednicki, Nucl. Phys. B238 (1984) 453 ;

H. Goldberg, Phys. Rev. Lett. 50 (1983) 1419;

J. Ellis, T. Falk, G. Ganis, K. Olive and M. Srednicki, Phys. Lett. B 510 (2001) 236, hep-ph/0102098.

[76] S. Heinemeyer, W. Hollik and G. Weiglein, JHEP 0006 (2000) 009, hep-ph/9909540. 
[77] ATLAS Collaboration, Detector and Physics Performance Technical Design Report, CERN/LHCC/99-15 (1999), see:

atlasinfo.cern.ch/Atlas/GROUPS/PHYSICS/TDR/access.html .

[78] A. Djouadi, Phys. Lett. B 435 (1998) 101, hep-ph/9806315

[79] G. Belanger, F. Boudjema and K. Sridhar, Nucl. Phys. B 568 (2000) 3, hep-ph/9904348;

G. Belanger, F. Boudjema, F. Donato, R. Godbole and S. Rosier-Lees, Nucl. Phys. B 581 (2000) 3, hep-ph/0002039.

[80] E. Richter-Was and D. Froidevaux, ATL-COM-PHYS-98-012.

[81] A. Belyaev, D. Garcia, J. Guasch and J. Sola, Phys. Rev. D 65 (2002) 031701, hep-ph/0105053.

[82] F. Maltoni, D. Rainwater and S. Willenbrock, Phys. Rev. D 66 (2002) 034022, hep-ph/0202205.

[83] M. Schumacher, talk given at the "Advanced Studies Institute, Physics at LHC", Prague, July 2003, see: asipraha.web.cern.ch/asipraha/2003/LHC/ .

[84] D. Zeppenfeld, R. Kinnunen, A. Nikitenko and E. Richter-Was, Phys. Rev. D 62 (2000) 013009, hep-ph/0002036.

[85] D. Rainwater and D. Zeppenfeld, Phys. Rev. D 60 (1999) 113004, E: ibid D 61 (2000) 099901, hep-ph/9906218;

D. Rainwater, hep-ph/9908378

N. Kauer, T. Plehn, D. Rainwater and D. Zeppenfeld, Phys. Lett. B 503 (2001) 113, hep-ph/0012351.

[86] T. Plehn, D. Rainwater and D. Zeppenfeld, Phys. Lett. B 454 (1999) 297, hep-ph/9902434.

[87] T. Plehn, D. Rainwater and D. Zeppenfeld, Phys. Rev. D 61 (2000) 093005, hep-ph/9911385.

[88] TESLA TDR Part 3: "Physics at an $e^{+} e^{-}$Linear Collider", eds. R. Heuer, D. Miller, F. Richard and P. Zerwas, hep-ph/0106315, see: tesla.desy.de/tdr .

[89] T. Abe et al. [American Linear Collider Working Group Collaboration], Resource book for Snowmass 2001, hep-ex/0106055, hep-ex/0106056, hep-ex/0106057, hep-ex/0106058.

[90] K. Abe et al. [ACFA Linear Collider Working Group Collaboration], hep-ph/0109166

[91] TESLA TDR Part 6, Chapter 1: "Photon collider at TESLA", hep-ex/0108012.

[92] V. Barger, M. Berger, J. Gunion and T. Han, Phys. Rept. 286 (1997) 1, hep-ph/9602415.

[93] C. Blöchinger et al. [Higgs factory working group of the ECFA-CERN study on Neutrino Factory \& Muon Storage Rings at CERN], "Physics opportunities at $\mu^{+} \mu^{-}$Higgs factories", hep-ph/0202199. 
[94] S. Heinemeyer and G. Weiglein, BNL-22-01.

[95] For a summary see: D. Zeppenfeld, in Proc. of the APS/DPF/DPB Summer Study on the Future of Particle Physics (Snowmass 2001) ed. R. Davidson and C. Quigg, hep-ph/0203123.

[96] For a summary see: H. Haber, hep-ph/9703381

[97] J. Brient, talk at the Linear Collider Workshop, Cracow, Poland, September 2001, see: webnt.physics.ox.ac.uk/lc/ecfadesy

[98] CMS Collaboration, see: cmsinfo.cern.ch/Welcome.html/CMSdocuments/CMSplots/ .

[99] T. Hahn, S. Heinemeyer and G. Weiglein, Nucl. Phys. Proc. Suppl. 116 (2003) 336, hep-ph/0211384.

[100] S. Dawson and S. Heinemeyer, Phys. Rev. D 66 (2002) 055002, hep-ph/0203067

[101] J. Erler, S. Heinemeyer, W. Hollik, G. Weiglein and P. Zerwas, Phys. Lett. B 486 (2000) 125, hep-ph/0005024

J. Erler and S. Heinemeyer, in Proc. of the 5th International Symposium on Radiative Corrections (RADCOR 2000) ed. H. Haber, hep-ph/0102083.

[102] D. Cavalli et al., [Les Houches Higgs working group], Summary report, hep-ph/0203056.

[103] G. Weiglein, hep-ph/0301111, see also: www.ippp.dur.ac.uk/ georg/lhclc/. 